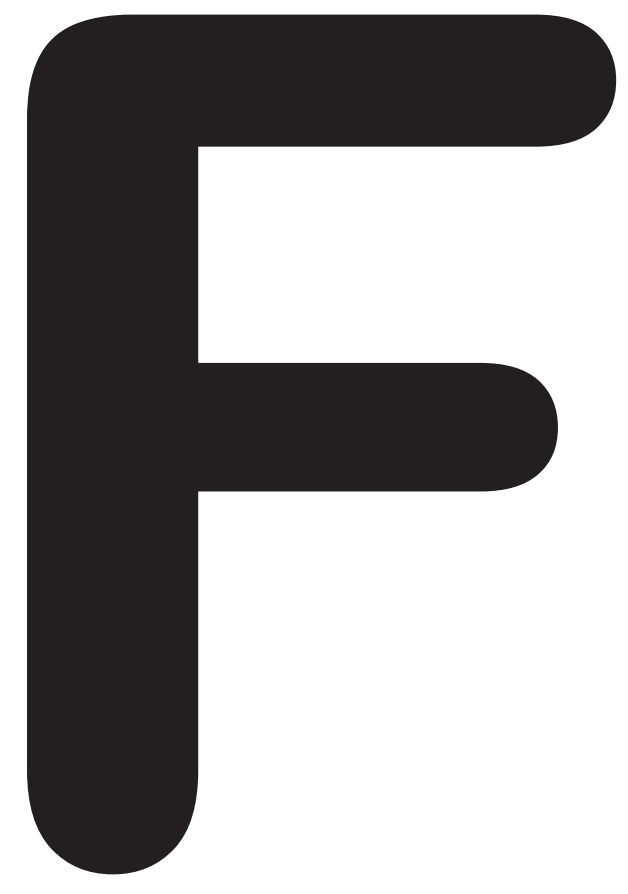

\title{
List of Authors and Co-Authors
}




$\overline{\mathbf{A}}$

Aarntzen E.H.J.G.: A-343

Aarts E.: $B-384$

Abbattista T.: $B-613$

Abdelmoez A.: B-554

AbdulQadhr G.: B-559

Abels B.: B-293

Aboud G.: A-159

Abrantes A.F.C.L.: B-443

Abro J.A.: B-494, B-497

Abulafi M.: B-526

Acharya R.U.: B-130

Acid S.: B-565

Ackermann H.: B-759

Adam G.: B-057, B-058, B-206, B-467, B-600, $B-620, B-623$

Adamietz B.: B-802

Adams J.E.: A-151

Adamsbaum C.: A-037

Adibi P.: $B-020$

Adjei-Gyamfi Y.: B-436

Afshan I.: B-608

Ahari C.: $B-603$

Ahlström H.: B-559

Ahmad I.: B-608

Ahmad M.E.: B-292

Ahn M.I.: B-275

Aida N.: B-476

Aigner F.: B-662

Aime S.: A-105, A-341

Akan K.: B-744

Akata D.: A-091, A-252

Akhadov T.A.: $B-838$

Akhlaghpoor S.: A-351, B-765

Akin O.: B-302, B-665

Akram A.R.: B-394

Al Bunni F: B-817

Al hazaimeh B.: $B-423$

Alanen K.: B-306

Albert J.: $B-694$

Albisinni U.: B-261

Alcolado Jaramillo A.B.: B-201

Alconchel A.: B-084

Alconchel J.J.: B-084

Aldhafeeri F.: B-519

Alejandre Lafont E.: B-640

Alemany M.: B-114

Alessandrino F.: B-703

Alessi S.: B-703

Alexiou E.: B-276

Alghamdi J.: B-519

Alibek S.: B-593

Alic L.: B-556, B-560

Aliprandi A.: B-864

Al-Islam S.: $B-743$

Alison M.: A-154, B-799

Alizai $\mathrm{H} .:$ : $B-172$

Alkadhi H.: B-382

Allen G.M.: A-309

Allmendinger T.: $B-148, B-368$

Almeida R.P.P.: B-443

Almén T.: B-707

Al-Nakshabandi N.A.: B-431

Alsaif H.S.: $B-389$

Alsop D.C.: B-531

Altenbernd J.-C.: B-144

Altman D.: B-330

Altmeyer K.: B-699

Alvarez-Linera J.: A-058

Amacker N.A.: B-854

Amann G.: B-745

Amato M.: B-146, B-279

Ames V.: B-801
Amet S.: B-230

Amoretti N.: B-281

Amoui M.: A-351

An J.: $B-495$

Anai H.: $B-133$

Andersen J.G.: B-446

Andreisek G.: B-570

Andresen R.: B-409

Andreu J.: A-192, A-365

Anfuso C.: $B-493$

Angelelli G.: B-192

Angeli E.: $B-150$

Angle J.F.: B-767

Angus J.-R.: SS MS 3

Anik Y.: B-515

Antal G.: B-579

Antiga L.: $B-481$

Antoch G.: A-045, B-118, B-144, B-486, B-535

Anzidei M.: B-242, B-343, B-349, B-488, B-491, $B-507, B-766, D-20$

Aoki T.: B-002

Apfaltrer P.: B-023

Apitzsch J.C.: B-128

Apprich S.R.: B-378

Aquaro G.D.: B-207

Arai O.: $B-804$

Araki T.: $B-138$

Arcadi T.: B-236, B-498

Arce P.: $B-773$

Arcidiacono P.: B-014

Ares J.V.: B-602

Argirò R.: B-277

Argyropoulou M.I.: A-035, A-205, B-661

Aribal E.: $A-387$

Arici V.: $B-703$

Arita J.: B-002

Armstrong A.M.: B-497

Armuzzi A.: B-439

Arnberg F.: B-111

Arndt M.: $B-430$

Arndt N.: B-794

Arnold R.: B-598, B-599

Arnoldi E.M.: B-238

Arns S.: $B-774$

Aronen H.J.: B-306

Arslan B.: B-767

Artho G.: B-526

Arya A.: B-198

Ascenti G.: B-493, B-791

Aschwanden M.: B-341

Ashoori N.: B-391

Aslam M.O.: B-431

Assael B.: B-625

Astrakas L.: A-229

Åström G.: B-559

Aswad R.: B-565

Attenberger U.I.: B-342, B-532

Atzwanger J.: $B-789$

Aubard Y.: B-068

Augart D.: B-711

Augustin M.: $B-250$

Aurilio G.: B-282

Autret G.: B-799

Avni F.E.: A-093

Awadalla M.M.A.: B-219

Ayaz U.Y.: $B-819$

Aydin H.: B-374, B-818

Aydingoz U.: A-312

Azevedo K.B.: B-443

Azevedo R.: B-539

Azevedo R.M.: B-619

Aziz Ahari A.: A-351, B-765

Aziza R.: $B-425$

Azizi A.: B-035, B-158, B-423, B-424

Azoulay R.: A-154

\author{
B \\ B. Joseph G.: B-172 \\ Baali A.: B-414 \\ Bacher K.: B-721, B-726 \\ Bachert P.: B-177 \\ Backes W.: B-169 \\ Backes W.H.: A-524, B-344, B-350 \\ Bae Y.J.: $B-499$ \\ Baer M.: B-363, B-584 \\ Baglio I.: $B-314$ \\ Bagni B.: $B-117$ \\ Bajic D.: $B-831$ \\ Bakers F.C.H.: B-165, B-169 \\ Bakhshayesh-Karam M.: B-627 \\ Bakic P.R.: A-214, A-495 \\ Balan K.K.: B-012 \\ Baldan E.: B-187 \\ Balériaux D.: A-411 \\ Ballesio L.: B-544 \\ Balleyguier C.S.: A-159, D-42 \\ Baloch E.: B-695 \\ Bals R.: B-393 \\ Baltzer P.A.: B-644, B-646 \\ Baltzer P.A.T.: A-433, B-318, B-319, B-487, B-549, \\ $B-550, B-642, B-643, B-679, B-680$
}

Balvay D.: B-799

Bamberg F.: B-232, B-243, B-411, B-492, B-695, $B-700, B-711, B-758, B-825$

Banckwitz R.: B-585

Bandirali M.: B-501, B-864

Bandula S.: $B-739$

Bankier A.A.: A-327

Banko B.: B-195

Bannas P.: B-057, B-467

Bansal M.: B-027

Baranes L.: B-850

Barbiani C.: B-333, B-496

Barbus V.: SS MS 2

Barentsz J.O.: A-401, A-423

Bargellini I.: A-148, B-153

Barikbin R.: B-020

Barile A.: A-143

Barillari M.: B-797

Barkhatova A.N.: B-838

Barkhausen J.: B-208, B-807, D-03

Barkhof $\mathrm{F}$ : : A-323, A-500

Barneveld Binkhuysen F.H.: A-536

Baron R.L.: A-360, A-400

Barone-Adesi L.: $B-687$

Baroud S.: B-129, B-844

Barraza J.M.: B-468, B-492, B-500

Barry de Longchamps N.: $B-310$

Barsi P.: A-412

Bartolozzi C.: B-123, B-136, B-153, B-207, B-222, $B-324, B-325, B-326, B-523, B-843$

Bashir M.S.R.: $B-855$

Basma S.: B-866

Ba-Ssalamah A.: A-012, B-129, B-844

Bastarrika G.: B-412

Bastati N.: B-844

Bastati-Huber N.: B-129

Båth M.: D-14

Batool M.: B-431

Battaglia V.: $B-153, B-843$

Battigelli L.: B-311, $B-312$

Battista G.: B-261, B-262

Bauer J.S.: B-180, B-263

Bauer R.W.: B-147, B-193, B-445, B-697, B-753, $B-755, B-759$

Bauer S.: $B-051$

Baum T.: B-172, B-263, B-264

Baumann M.: B-653

Baumann T.: B-018, B-609 
Baumeister R.G.: B-681

Bäumer P.: B-214

Bauner K.: B-411

Bauner K.U.: B-097, B-210

Baxa J.: B-296, $B-793$

Bazot M.: B-796

Bazzocchi A.: B-261, B-262

Bazzocchi M.: B-072, B-311, B-312, B-315

Beale T.: $A-491$

Becce F.: B-427, B-568

Beck M.: B-378

Beck Y.: B-002

Becker A.: B-391, B-411, B-695, B-779

Becker C.: B-232, B-345, B-684, B-700, B-772, $B-825$

Becker C.D.: A-189

Becker H.-C.: A-005, B-711

Becker H.-C.R.: B-758

Becker M.: A-493, B-772

Becker-Lienau J.: B-401, B-407

Beer A.: $B-100, B-419$

Beer A.J.: B-113, B-305, B-421

Beets G.L.: B-161, B-163, B-165, B-167, B-169, $B-528$

Beets-Tan R.G.H.: A-479, A-504, B-161, B-163, $B-165, B-167, B-169, B-528$

Beheshti M.: A-019

Behr J.: B-391

Beigelman C.: A-474

Belblidia A.: B-769

Belgour A.: B-415

Belgrano M.: B-686

Bell D: : B-335

Bellemann N.: B-260

Belli A.-M.: $A-176, A-211$

Belli G.: B-482, B-483, B-485, B-865

Bellin M.-F.: A-197

Bellomi M.: B-009, B-025, B-282, B-481, B-675

Belsan T.: B-066

Beltran L.S.: B-176

Bemi P.: B-326

BenDriss A.: B-093

Bendszus M.: $B-214$

Beningfield S.: $B-371$

Benjamin-Laing $\mathrm{H}$.: $B-743$

Benndorf M.: B-487, B-679

Bérczi V.: $A-177, B-241$

Beregi J.-P.: $A-118$

Berends F.J.: B-384

Berger F.: A-208

Bergner F.: $B-367$

Berjano E.: B-038

Berkhof M.: B-161

Bernard M.: B-417

Bernardini A.: B-095, B-099

Bernardo S.: B-693

Berndorf M.: SS MS 1

Bernhardt P.: B-091

Bernsen M.: B-560

Berritto D.: B-654, B-655

Berry I.: $B-043$

Bertocchini A.: B-734

Bertolini M.: B-589

Bertolo S.: B-624, B-625

Bertolotto M.: A-260, B-081, B-686

Bertomeu M.: B-114

Bertrand D.: B-231

Bertugno S.: $B-202$

Besostri V.: B-055

Besutti G.: B-615, B-617

Betge S.: $B-221$

Bettelheim D.: $B-471$

Betz M.: B-100

Beyer H.-K.: B-267
Beyer T.: A-004

Bezzi M.: B-277

Bezzon E.: B-187

Bhagat N.: $B-156$

Bhalla A.: B-254

Bhalla M.: $B-638$

Bhalla N.: $B-638$

Bhasin D.: B-254

Bhatnagar P.: B-194

Bhatti W.: B-743

Bhopal U.: $B-431$

Bi J.: $B-760$

Bi X.: B-248

Biagini E.: B-203

Bianco V.: $B-489$

Bianda N.: B-247

Bidault F.: A-159

Biederer J.: $B-148$

Bieri O.: B-096, B-178

Bierry G.: B-606

Biesheuvel C.: B-182

Biffar A.: B-097

Bilbao J.I.: A-210, A-512

Bilecen D.: $B-341$

Billette de Villemeur T.: $B-835$

Billiard J.-S.: B-769

Billing $\mathrm{H} .:$ A-490

Binkert C.: A-306

Binukrishnan S.: B-708

Biondi T.: $B-301$

Bipat S.: B-323, B-652

Bischof A.: $B-807$

Bischoff P.: B-208, B-807

Bisdas S.: A-008, A-086, B-216

Bisogno R.: B-240

Bittersohl B.: B-378, B-601

Bittner M.: $B-832$

Bize P.: $B-427$

Bjorndal H.: B-186

Bladowska J.: $B-213$

Blandino A.: B-791

Blanke P.: B-026, B-088, B-609, B-823

Bleeker M.C.G.: B-529, B-795

Bleeser F.: B-726

Bley T.A.: $B-688$

Blijd J.: $B-156$

Block T.: $B-849$

Bloem J.L.: A-514

Blomqvist L.: $B-735$

Blomqvist L.C.O.: A-478

Blondiaux E.: B-835

Blondin D.: B-248

Bluekens A.M.J.: B-181

Blum A.: $D-12$

Boachie C.: B-183

Bobek-Billewicz B.: B-104

Bochmann K.: B-355

Bock C.: B-102

Bock E.: B-439

Bock H.C.: B-105

Bock K.: B-037

Bock M.: A-335

Böckler D.: B-633

Boeckler D.: $B-867$

Boekstegers P.: B-700, B-825

Boerman O.C.: $A-343$

Boermeester M.A.: B-652

Boetes C.: A-432, B-184

Bogdan M.: B-644, B-646

Böhm M.: B-393

Bohndorf K.: A-022, B-379

Boi C.: $B-504$

Boi L.: B-404, B-405, B-408, B-460

Boijsen M.: D-14
Bol K.: B-560

Bonaffini P.: $B-252$

Bonaffini P.A.: B-056, B-253

Bonastre J.: $B-425$

Bonello L.: B-009, B-231, B-481, B-675

Bonetti F.: B-314, B-316

Bonetto F.: $A-343$

Bongartz G.: $B-341$

Bonomo L.: A-379, B-048, B-063, B-094, B-095, B-099, $B-146, B-279, B-439, B-477, B-685, B-687$

Booij R.: $B-860$

Boone C.: B-517

Boone D.J.: B-329, B-330

Boone J.M.: A-133

Boraschi P.: B-136, B-222

Borberg T.: $B-807$

Borges A.: A-085

Boric I.: A-180

Borlak J.: $B-778$

Borra R.J.: B-306

Bosanac D.: B-612

Bosari S.: $B-525$

Bosmans H.: A-182, A-238, A-496, B-641, B-726

Boss A.: B-536

Bossuyt P.M.M.: B-652

Botar-Jid C.: B-682

Botnar R.: B-552

Bots M.L.: B-830

Bottari A.: B-346, B-493

Boudreau R.: $B-787$

Bouillet P.: B-068

Boulenguez C.: $B-274$

Boulet B.: A-159

Boulos P.: B-522

Bourguet P.: A-055

Bouzian N.: B-226, B-227

Bowers D.C.: $B-837$

Bowtell R.W.: A-337

Boyd L.: A-220

Boyer B.: A-159

Boyer L.: B-762

Boyes S.: $B-426$

Bozzao A.: B-108

Bozzi E.: $B-843$

Bracard S.: A-435, B-456

Brade J.: B-199

Brader P.: $A-270$

Brambilla G.: B-047

Brambs H.-J.: B-022

Brancatelli G.: A-301, A-454

Brandalise A.: $B-316$

Braren R.: B-113, B-552

Brauer T.: $B-155$

Braunschweig T.: $B-128$

Breatnach É.: A-055

Brehm M.: B-367

Bremer C.: B-779

Bremerich J.: B-096

Brennan C.: B-718

Brian D.: $B-338$

Briganti C.: $B-520$

Briggs R.H.: $B-527$

Brillet E.: B-054

Brkljacic B.: A-258, A-459, B-077

Brockmann C.: B-300

Broeders M.J.M.: B-181

Brook A.: B-256

Brook O.R.: B-256

Brophy D.P.: B-870

Bros S.: $B-762$

Brosche C.: B-155

Brountzos E.: B-503

Brown C.: $B-469$

Brown G.: A-476, B-526 
Brückmann $\mathrm{H} .:$ B-588

Bruder H.: B-361, B-362, B-368

Brugger P.C.: B-471, B-472, B-800, B-832, B-833, B-834

Brummund L.: $B-856$

Brunelli E.: $B-613$

Bruners P.: $B-749$

Brunetti N.: $B-236$

Bruneval P.: $B-226$

Bryan L.: B-335

Bryant M.G.H.: B-278

BS A.: $B-284$

Buchbender C.: B-601

Buchheidt D.: B-607

Buck A.K.: $A-017$

Bücker A.: B-699

Budde R.P.J.: $B-824$

Buecker A.: B-393, B-406, B-632, B-690

Buerk J.: $B-026$

Buerke B.: $B-647$

Bühlow R.: $B-131$

Bulla S.: B-026, B-088, B-609, B-823

Buls N.: $B-851$

Buonocore V.: B-301, B-308, B-666

Bura R.: $B-242, B-244$

Burdío F.: $B-038$

Burghardt A.J.: B-264

Burgmans M.C.: B-152

Burke M.: $B-588$

Burling D.: A-075

Busard M.P.H.: B-529, B-795

Busetto G.: $B-308$

Busilacchi P.: B-613

Busoni S.: B-865

\section{C}

Cabibbo B.: B-001

Cabrera M.N.: B-115

Cacaci S.: A-129

Cáceres J.: A-023, A-128, A-132, A-245, A-361, A-501

Cademartiri F.: A-425, B-236, B-498

Cakal E.: B-819

Cakir E.: $B-819$

Calabrese M.: $B-677$

Calabrò E.: B-028

Calandrelli R.: $B-477$

Calandriello L.: B-146

Calbo J.: B-813

Caliari G.: $B-496$

Calli C.: A-392

Calliada F.: $B-703$

Caloggero S.: B-346, B-791

Camara O.: $B-550$

Camera L.: B-314, B-316

Campana L.: $B-781$

Campanella D.: $B-327$

Campani D.: B-843

Campos G.C.: $B-583$

Camps Herrero J.: A-046, A-352, B-317

Cañadas M.: $B-773$

Canale S.: A-159

Cancian L.: $B-137$

Candusso M.: $B-732$

Canini R.: B-261, B-262

Cano Gimeno J.: B-317

Cano J.: B-154

Cantin S.: $B-511, B-512$

Cantisani V.: $B-388, B-815, D-30$

Cantwell C.P.: $B-870$

Canu T.: B-205, B-781

Cao H.: B-145

Cao K.: B-016, B-798
Cao Y.: B-299

Capodiferro S.: B-192

Cappabianca S.: B-655

Cappendijk V.C.: B-163, B-165, B-169, B-528

Capraro C.: B-021, B-119, B-162

Capuano E.: $B-498$

Caramella D.: A-488, A-535

Carballido-Gamio J.: $B-172$

Carbognin G.: B-314, B-316

Carbonaro L.A.: B-541

Carbone I.: B-202

Cardaioli G.: B-241

Cardoen L.: B-017

Cardoso M.M.: B-692

Carels K.: $B-017$

Carminati M.: $B-828$

Caroli A.: $B-481$

Carrafiello G.: B-631, B-634

Carrai P.: B-153

Carreras J.L.: B-115

Carrillo M.C.: B-629

Carstairs C.: SS MS 1

Carter R.: $B-743$

Cartocci G.: B-049, B-343, B-349, B-491, B-507, $B-766$

Casali V.: $B-544, B-546, B-676, B-678$

Caseiro-Alves F.: $A-353$

Cassagnes L.: $B-762$

Cassone V.: $B-346$

Castañer E.: A-502

Castellón Plaza D.: B-233

Castot A.: B-230

Castro M.C.: $B-813$

Catalano C.: B-005, B-049, B-343, B-349, B-488, $B-491, B-507, B-544, B-546, B-624, B-676$, $B-678, B-766, D-36$

Catane R.: $B-672$

Catarino A.: B-572

Catena N.: $B-479$

Caulo A.: B-146, B-381

Caulo M.: $B-520$

Caussin C.: $B-231$

Cava M.: B-205

Cavaliere C.: B-654, B-655

Cavallaro A.: B-124

Cavallaro M.: B-081, B-166

Cavallo Marincola B.: B-343

Cavero Guibanel M.A.: B-201

Cazejust J.: A-034

Cederberg K.: $B-837$

Cejna M.: B-402

Cellina M.: B-014, B-386, B-462

Cento E.: B-397

Cercueil J.-P.: B-750, B-764

Ceretti Pisani A.: B-525

Cerezal L.: $A-030$

Cerri F.: $B-325$

Cevener M.: B-768

Chabbert V.: $B-231$

Chabrot P.: B-762

Chai W.: B-618

Chai W.M.: B-514

Chakraborty S.: B-292

Chalard F.: $B-835$

Chalazonitis A.: B-298

Chalouhi G.E.: B-799

Champsaur P.: B-565

Chan N.: B-715

Chandelier F.A.R.: B-121

Chang C.-Y.: B-683

Chang I.S.: B-639

Chapman S.: A-038

Charimo Torrente J.: $B-751$

Chateau F.: B-116
Chateil J.-F.: A-405

Chatellier G.: B-395

Chaterjee F.: B-436

Chatzikonstantinou A.: $B-857$

Chau M.: B-043, B-414

Chedia S.: $B-212$

Chellamuthu S.: B-027

Chemla D.: $B-420$

Chen B.: B-760

Chen F: : B-551

Chen G.: B-008

Chen J.: B-042

Chen K.: B-010, B-618

Chen K.M.: B-514

Chen T.W.: B-011

Chen Z.: B-508

Cheng $\mathrm{H}$.: $B-098$

Cheng Z.: B-596, B-826

Cherot-Kornobis N.: $B-274$

Cherqui D.: $B-850$

Chersevani R.: $B-187$

Chhabra A.: B-570

Chiantera V.: $A-458$

Chiaramida S.A.: B-334

Chiaravallotti A.: B-460

Chiesa F.: $B-677$

Chiesa G.: B-150

Ching A.S.-C.: B-557

Chiorean A.: B-682

Chipon E.: B-512

Chiti A.: A-057

Chiu W.W.Y.: B-743

Chmeissani M.: B-773

Cho B.-S.: B-410

Cho E.-S.: B-712, B-792, B-847

Cho K.R.: B-547, B-548

Choi B.I.: B-015, B-168, B-251, B-611, B-842, B-849

Choi H.Y.: $B-674$

Choi J.W.: $B-822$

Choi J.-Y.: B-004

Choi M.: B-275

Choi S.I.: B-499

Choi S.Y.: $B-410$

Chong V.: $A-223$

Choukroun G.: $B-230$

Chow P.K.H.: B-152

Chowdhury F.U.: B-194, B-527

Christiaens L.: B-231

Chu Z.-G.: $B-461$

Chuck N.C.: B-536

Chun E.J.: B-499

Chung A.Y.F.: B-151

Churkina S.: B-069, B-070

Cianci R.: B-489

Cibere J.: B-379

Ciccarone A.: B-731

Cicciò G.: B-493

Cicio G.: B-373

Cicorelli A.: $B-153$

Ciet P.: $B-624, B-625$

Cikara I.: B-077

Cina A.: $B-687$

Cini L.: $B-326$

Ciolina F.: $B-766$

Cioni R.: B-153

Cipriani A.: B-687

Cira K.: B-768

Ciresa M.: B-146

Ciuce C.: B-682

Ciurea A.: B-682

Claudon M: : A-207

Clausen M.: B-467

Clavien P.-A.: B-382, B-385

Clemens K.: B-706 
Clément O.: A-102, B-230, B-701, B-799

Clemente A.: B-236

Clementi E.: B-205

Cleutjens J.: $B-184$

Clevert D.-A.: B-050, B-074, B-246, B-684, D-43

Climent F.: $B-217$

Clusmann $\mathrm{H}$.: $B-451$

Cobbold J.F.L.: A-385

Cockmartin L.: B-641

Cohnen J.: B-105

Colagrande S.: B-482, B-483, B-485

Cole E.: B-548

Colicchio G.: B-520

Cologna A.J.: B-669

Colombo M.: B-162

Colombo P.: B-590

Colosimo C.: B-477, B-520

Colosimo M.: B-634

Colsher J.: $B-855$

Combaz X.: A-230

Combe C.: $B-540$

Comelli S.: $B-452$

Conte D.: B-277

Contegiacomo A.: B-279

Conzelmann L.: $B-041$

Coolen J.: A-280

Cooper S.L.: B-218

Copley S.J.: A-525

Corcione S.: B-671

Corkill R.A.: B-747

Cornalba G.: B-047, B-240, B-525

Cornud F.: A-421, B-310

Corot C.: B-226, B-227

Correale L.: $B-327$

Correas J.-M.: A-395, B-814, D-27, D-37

Corso R.: B-252, B-253

Cortesi E.: $B-277$

Corti R.: B-247

Cosín O.: $B-154$

Costantini A.: B-477

Costanzo S.: $B-381$

Costello P.: B-412, B-494, B-709

Cotroneo A.R.: $B-489$

Cotta E.: B-634

Cotten A.: A-195, A-324

Couchon S.: $B-395$

Coudray C.: B-796

Coumans J.: $D-05$

Coutouly X:: B-670

Coutroubis D.: $B-013$

Cova L.: B-290

Cova M.A.: B-001, B-081, B-166, B-686

Cowan N.C.: A-198

Cowling C.: A-220

Cox K.: B-071

Cox M.G.P.J.: $B-830$

Coyle J.: B-718, $B-727$

Cozzone P.: $B-417$

Cramer M.J.: $B-830$

Cramer M.J.M.: B-340

Crean A.: B-337

Crema M.D.: $B-171, B-174$

Cremers P.T.J.: $B-524$

Cremers S.E.H.: B-524

Crespo C.: $B-813$

Creuze N.: $B-420$

Cristel G.: B-673

Crivellaro M.: B-055

Crochet D.: $B-231$

Crystal P.: B-313

Csóka M.: $B-738$

Cucca M.: $B-117$

Cuesta M.A.: B-795

Cugini $C$.: $B-137$
Cui Y.: B-016, B-798, B-816

Cuinet M.: $B-425$

Cullmann J.: $B-751$

Cunha M.L.: B-583

Cunningham P.M.: A-463

Curati-Alasonatti W.: A-369

Curigliano G.: B-675

Cyran C.C.: B-464, B-553, B-780

Czech T.: B-471

Czerny C.: A-060

$\overline{\text { D }}$

da Cunha $\mathrm{G}: \mathrm{B}$ :

Da Ros V.: $B-405$

Dacher J.-N.: B-345

d'Adamo E.A.: B-277

D'Agostino E.: $B-726$

Dai Q.: B-495

Dai Y.: $B-145, B-360$

Dal Col A.: B-072, B-311

Dale B.M.: B-619

Dalke D.B.D.Z.: SS MS 3

Dallaudiere B.: B-059

Daltro P.A.: A-234, B-692

Damilakis J.: A-290, B-321, B-648

Dance D.R.: $B-723$

D'Angelo G.: B-205

Danielsson M.: B-185

Danza F.M.: B-064, B-439

Darai E.: B-796

d'Archambeau O.: B-458

Darchis C.: $B-757$

Darge K.: $B-736$

D'Argento $\mathrm{F}: \mathrm{B} B-732, B-734$

Darnell A.: A-237

Darrasse L.: A-103

Darvishi Nakhl Ebrahimi A.: B-424

Dattesi R.: B-048, B-685

Daubiné F.: $B-226, B-227$

Daurès J.-P.: $B-237$

David V.: B-204, B-663

Davishi A.: B-035

Davishi Nakhl Ebrahimi A.: B-158

De Belder F.: A-295, A-436, A-523

de Bock G.H.: B-280

De Bucourt M.: B-509

de Campos R.: B-539

de Campos R.O.P.: B-619

De Cecco C.: B-202

De Cecco C.N.: B-204, B-663

De Cobelli F.: B-014, B-202, B-205, B-673, B-781

De Coninck T.: $B-785, B-788$

De Crop A.: $B-721$

de Feyter P.J.: $B-416$

De Filippis G.: B-005

De Franco A.: B-439

de Haan M.C.: $B-323$

de Herder W.W.: B-812

de Hoop B.: B-392

De lorgi V.: $B-673$

de Jong M.: B-560

de Jong M.D.F.: B-075

de Kerviler E.: A-079, B-229

de Lange C.E.: $B-473$

De Malherbe M.: $B-752$

De Man B.: B-364

De Meerleer G.O.: B-307

De Mey J.: B-345, B-851, D-39

De Ponti E.: $B-162$

de Roos A.: A-381

De Ville de Goyet J.: B-732, B-734
De Visschere P.J.: B-307

De Vleeschouwer S.: B-101

de Vries I.J.M.: A-343

De Wever W.: A-280

De Wever W.F.M.: A-526

De Wilde J.: A-292

De Zordo T.: B-662

Deak P.: B-593

Dean K.: B-306

Debbage P.: B-554

Decaux O.: B-054

Dechoux S.: B-555

Decker T.: $B-182$

Declerck C.H.: B-517

Degani H.: B-672

Degenhart C.: B-401

Dekker H.M.: B-705

del Ciello A.: B-146, B-279

Del Giudice C.: $B-408$

Del Maschio A.: B-014, B-205, B-386, B-462, $B-673, B-781$

Delaloge S.: $A-159$

Delank K.-S.: $B-267$

Delgado I.: B-475

Delgado Sanchez-Gracián C.: B-233

Della Pina C.: A-209

Della Vigna P.: $B-282$

Dellabianca C.A.: B-703

Delles M.: $B-045$

Delmas Y.: $B-540$

Delorme S.: $B-135$

Demaerel P.: B-101, B-355

Deminière $C$.: $B-540$

Demirci A.: B-515

den Heeten G.J.: B-181

Deng D.M.: B-060

Denys A.: $B-427$

Deray G.: $B-230$

Derchi L.E.: $A-185, A-259$

Derieppe M.: $B-540$

Derlin T.: B-058, B-467

Deroo D.: $B-865$

D'Errico L.: $B-207$

Deschamps F.: A-219, A-417, D-35

Deseive S.: B-697

Deshpande R.: B-106

Deuerling-Zheng Y.: B-455

Deutschmann H.: B-250

Deutschmann J.: B-127

Deutschmann J.H.: B-179

Deux J.-F.: $B-231$

Devalia H.: $B-071$

Devaraj A.: $B-272$

Devauchelle A.-D.: B-456

Deveaux V.: $B-555$

Deveraj A.: B-271

Devriendt D.: $B-017$

Dewachter P.: B-701

Deylgat B.: B-017

Dezortová M.: A-344

Dhapare A.C.: B-616

D'Herde K.: B-721

Dhouib Chargui A.: B-835

Diakatou V.: SS MS 2

Di Bella G.: B-207

Di Egidio V.: $B-353$

Di Filippi R.: $B-481, B-675$

Di Giovanna E.: $B-103$

Di Girolamo M.: B-204, B-663

Di Grezia G.: $B-285$

Di Lelio A.: B-055, B-056

Di Lella G.M.: $B-477$

Di Leo G.: B-047, B-240, B-525, B-541, B-828

di Maggio $C .: B-187$ 
Di Mare L.: B-049, B-343, B-349, B-491, B-507, B-766 Di Martino M.: B-005, $B-488$

Di Massa A.: B-634

Di Maurizio M.: $B-731$

Di Miscio R.: B-005, B-488

Di Molfetta V.I.: $B-094$

Di Paolo P.L.: B-049

Di Pisa G.: B-021

Di Segni M.: $B-815$

Di Valentino M.: $B-247$

Di Veronica A.: $B-439$

Dichgans M.: B-243, B-464

Dicken V.: $B-647$

Diederich S.: A-217

Diedrichsen L.: $B-853$

Dietemann J.-L.: B-606

Dietrich O.: $B-176, B-553, B-780$

Dietrich T.J.: $B-377$

Dietzel A.: B-644, B-646

Dietzel M.: A-433, B-318, B-319, B-487, B-549, $B-550, B-642, B-643, B-644, B-646, B-679$, $B-680$

Dijkshoorn M.: B-696

Dijkshoorn M.L.: B-592, B-860

Dilli A.: B-819

Dillmann R.: B-045

Dimostheni A.: $B-216$

Ding J.: $B-249, B-714$

Dinkel J.: $B-148$

Dinter D.: $B-607$

Dittrich E.: $B-832$

Djabbari M.: B-850

Dobritz M.: B-234, B-263, B-348, B-861

Doelken M.: $B-455$

Doerfler A.: B-455

Doleschel D.: $B-774$

Dolgun A.: B-211

Domayer S.: $B-790$

Domingues R.C.: B-692

Donati F.: B-136, B-222

Donati O.: $B-536$

Donato R.: B-493

Dong S.: $B-157$

Dong Z.-H.: B-558

Donker H.C.W.: B-653

Donoso L.: A-487

Dora C.: $B-377$

Dorn F.: B-234, B-291, B-603

Dousset V.: A-122

Dowlatshahi D.: $B-292$

Drapé J.-L.: A-028, B-568

Drechsler K.: B-124

Dremmen M.H.G.: B-087

Dromain C.: A-159, D-15

Drosos A.: B-276

Du Y.S.: B-235

Dubelaar I.J.M.: B-075

Düber C.: B-041

Ducou le Pointe H.: B-835

Dudea S.M.: $B-682$

Duhamel A.: B-274

Duijm L.E.M.: $B-805, B-806$

Dumitriu D.: $B-682$

Dumousset E.: $B-762$

Duncker D.J.: B-696

Dunet V.: $B-428$

Duning T.: $B-295$

Durakovic N.: $B-402$

Durmus T.: $B-821$

Duschka R.L.: B-208

Duvauferrier R.: B-054

Dyker K.: B-194

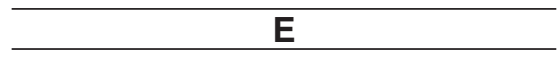

Ebdon-Jackson S.: A-403

Eberhardt R.: $B-278$

Ebner F:: B-250

Eckstein F.: B-180

Edme J.-L.: B-274

Efendi $\mathrm{H}$ :: $B-515$

Efremidis S.C.: $A-455$

Eftimov L.: $B-711$

Eggebrecht H.: B-694

Eggermont A.: $B-556$

Eggesbø H.B.: A-289

Eiber M.: B-305, B-376, B-484

Eichfeld U.: B-149

Eichinger M.: B-626

Eide K.R.: A-443

Eigenheer S.: $B-538$

Einarsdóttir A.: A-396

Eisenblaetter M.: B-779

Eisenhuber E.: A-166

Eiss D.: $B-310$

Eker O.: $B-511$

Ekseth U.: B-186

Elalfy M.: $B-778$

Elias R.: B-812

Elias-Jr J.: B-669

Ellegast R.: $B-786$

Ellestad M.: B-586

Elliot C.: B-400

Elliott S.T.: $D-28$

Elmståhl B.: B-707

Elsabaie M.: $B-424$

Elsiofy N.: $B-219$

Emad Y.: B-605

Emonds G.: $B-517$

Emsell L.: $B-513$

Engel A.: $B-398$

Engelen S.M.E.: B-167

Engelhorn T:: $B-455$

Englund $M$ : : $B-174$

Enriquez G.: A-203, B-475

Eo $\mathrm{H} .: \mathrm{B}-836$

Eppenberger P.: B-570

Erb G.: B-102

Erbel R.: B-694

Erbì V.: $B-504$

Erdt M.: $B-124$

Erfurt C.: $B-480$

Ernemann U.: B-216

Espeland A.: B-449

Esposito A.: B-205, B-781

Esposito M.: $B-731$

Esseridou A.: $B-864$

Essig M.: A-165, B-110, B-719

Esteban A.: B-602

Esteban Sanchez J.: B-086

Esteban-Hernández E.: B-154

Etezazian S.: B-020

Eustace J.: $B-727$

Eustace S.J.: A-144, A-376

Evert M: $B-383$

Eyal E.: B-672

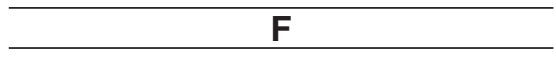

Faa G.: B-245, B-357

Fabbri A.: $B-650$

Fabbro E.: $B-742$

Faccioli N.: $B-134, B-797$

Faedda C.: B-677

Fagerberg U.L.: $B-735$

Faggioni L.: B-123, B-324, B-325, B-326, B-523

Fairbairn K.J.: $B-566$

Faivre J.-B.: $B-752, B-756, B-757$

Faivre-Pierret M.: $B-671$

Falaschi F.: B-136, B-222

Falco P.: B-327

Fan L.: $B-141$

Fan Z: : B-470

Fanariotis M.: $B-276$

Fanelli F.: B-403

Farghadani M.: $B-020$

Farghal A.S.: $B-433, B-434$

Farina D.: A-288, B-196

Farrell C.: $B-708$

Farrell M.A.: A-147

Farshid P.: B-035, B-158, B-423, B-424

Fassaert T.A.: B-075

Fatehi M.: A-351

Fattori R.: B-202, B-203

Fausto A.: B-650

Favat M.: $B-345$

Favia G.: B-192

Fedele I.: $B-673$

Federica C.: $B-166$

Federle M.P.: $A-445$

Fedorova A.: B-070

Fegeler C.: $B-867$

Fei M.: B-438

Feinberg Shapiro S.M.: B-672

Feldman L.: B-093

Felson D.T.: $B-171, B-174$

Feng S.-T.: $B-557$

Feng X.Y.: $B-220$

Ferda J.: $A-032, B-296, B-793$

Ferdova E.: B-296, B-793

Fernandes E.: B-572

Fernández Martín C.: B-086

Ferrandina G.: B-064

Ferrara F.: B-099

Ferrari M.D.: $B-351, B-352$

Ferrero G.: B-372, B-373, B-742

Ferrer-Puchol M.D.: B-154

Ferretti G.R.: A-368

Ferro F: : B-137

Ferrucci J.: $B-283$

Feuchtner G.: A-484

Feuerlein S.: B-022

Fewins $\mathrm{H} .:$ : $B-708$

Feyen L.: B-294, B-295

Fiaschetti V.: $B-657$

Fierro F.: B-693

Fierro S.: $B-204$

Figdor C.G.: A-343

Figueira J.C.: $B-621, B-622$

Filimonow S.: $B-078$

Filippiadis D.K.: $B-503, B-506$

Filippone A.: B-489

Filipponi F.: $B-153, B-222$

Fingerle A.: $B-861$

Fingerle A.A.: $B-348, B-484$

Fink C.: $B-023, B-199, B-334, B-413, B-597$, $B-857$

Fintha A.: B-594

Fiocchi F: $B-117, B-615$

Fiorina P.: $B-386, B-462$

Firouznia K.: $A-347$ 
Fischer A.-C.: A-179

Fischer L.: $B-127$

Fischer M.A.F.: B-382, B-385

Fischer R.: $B-206$

Fischer S.: $B-430$

Fischer T.: $B-078, D-23, D-31$

Fish D.: B-071

Fishman E.K.: A-354

Fitzgerald P.: B-469

Flacke S.: $B-451$

Flamini R.C.: $B-583$

Flavian A.: B-417

Fleischmann C.: B-369

Flinck A.: $D-14$

Flohr T.: B-361, B-362, B-368, B-585

Flohr T.G.: $B-582$

Flor N.: $B-240, B-525$

Flotats M.: $B-475$

Fonda C.: $B-731$

Fontaine A.: $B-281$

Fontana F.: B-631

Foreman J.: B-801

Forment M.: B-154

Forment Navarro M.: $B-317$

Fornage B.D.: A-321

Forsting M.: $B-144, B-535$

Forstner R.: A-039, A-505, B-789

Foster K.: $A-130$

Foti G.: B-134, B-797

Fraioli F.: $B-625$

Francès $C$.: $B-230$

Franchi P.: $B-279$

Francone M.: A-441

Franiel T.: $B-302$

Franke P.: B-599

Franklin J.M.: $B-399$

Franquet T.: A-077, A-282

Fransen $\mathrm{H}$.: $B-458$

Frantzen M.J.: B-740

Frascarolo P.: $B-427$

Fratta Pasini A.: $B-496$

Frauenfelder T: $B-854$

Frauscher F.: $B-662$

Freeman A.H.: A-184, A-254

Freilinger T.: $B-243$

Freling N.J.M.: A-298, B-392

Frellesen C.: $B-759$

Fretellier N.: B-226, B-227

Frey S.: B-199

Friedrich C.: B-085, B-440

Friedrich N.: $B-383$

Fries P.: B-632, B-690, B-699

Frija G.: $B-395$

Frisch M.: B-600

Froehlich J.M.: B-538

Frölich A.M.J.: B-359

Frotscher C.: $B-184$

Fugazzola C.: B-631, B-634

Fujii H.: $B-776$

Fujii K.: $B-804$

Fujii M.: $B-820$

Fujimoto K.: $B-543, D-26$

Fujisawa M.: $B-820$

Fukushi M.: $B-776$

Fukutomi T.: B-804

Fulcheri C.: $B-865$

Funari M.B.G.: B-583

Funel V.: $B-657$

Funicelli L.: $B-025$

Funovics M.A.: A-375, A-510

Funovics P.: $B-745$

Furman-Haran E.: B-672

Furtner J.: B-215

Fusaro F.: $B-452$
Fuschi M.: B-353

Fuser M.: $B-137$

Fuzzi F.: B-262

$\overline{\mathbf{G}}$

Gaa J.: B-484

Gaballa A.: B-053, B-561

Gabata T.: B-255

Gaeta M.: B-791

Gagliardo C.: B-103

Gagua M.: B-212

Gaillard S.: B-229

Gajda M.: B-549, B-550, B-643

Galanski M.: B-537, B-778

Galassi S.: B-663

Galati F.: B-301, B-308, B-666

Galatola G.: $B-327$

Gale A.: B-330

Gale A.G.: $A-448$

Galiano K.: B-287, B-717

Galimberti S.: B-061

Gallino A.: B-247

Gallot D.: B-762

Gallucci M.: A-522

Gamanagatti S.: $B-132$

Gan C.L.: B-748

Gandini R.: B-404, B-405, B-408, B-460

Ganeshan B.: B-013

Gangi A.: A-464

Ganten M.: B-135

Ganter C.: B-421

Gao F.: $B-558$

Gao P.: B-839

Gao S.-Y.: B-798

Garami Z.: $B-241$

Garant A.: B-526

García M.: B-115

Garcia Pavia P.: B-201

García-Peña M.P.: A-340

Garel C.: A-235, B-835

Garg P.: B-132

Gargan L.: $B-837$

Gärtner F.C.: $A-116$

Gaskarth M.T.G.: A-319

Gasparetto T.D.: B-692

Gatta G.: B-285

Gaudino S.: $B-477$

Gaxotte V.: B-093, B-209

Gazeau F.: B-555

Gazhonova V.: B-069, B-070

Ge Y: : B-347

Gebregziabher M.: B-494

Geddie W.: B-286

Geenen R.W.F.: A-114

Geeraert N.: B-724

Geiger D.: $B-005$

Geiger J.: B-598, B-599, B-688

Geijer H.: B-707

Geisthoff U.W.: B-690

Genisson J.-L.: B-540

Gennari F.: B-732, B-734

Gennaro G.: $B-187, D-13$

Gentinetta F.: $B-150$

Gerigk L.: B-110, B-719

Geschwind J.F.: B-156

Ghanaati H.: A-346

Ghazavi A.: B-020

Ghofrani M.: B-627

Ghysels M.: A-246

Giammarile F.: B-116

Giannakis D.: B-661

Giannoni M.F.: B-241
Giardino A.: B-828

Giarraputo L.: B-001

Gielen J.L.M.A.: A-416

Giesel F.L.: B-633, B-706

Giganti F.: $B-014$

Gigoni R.: $B-136, B-222$

Gilbert F.J.: A-434, B-183

Gilhuijs K.: A-239

Gillaizeau F.: $B-701$

Gille H.: $B-113$

Gilligan P.: A-404

Gimi B.: $B-837$

Ginocchi V.: B-589

Girard N.: A-230

Girardi V.: B-314, B-316

Girman P.: A-344

Gjesdal K.-I.: $B-542$

Glaser C.: A-255, B-176, B-534, B-567, B-786

Glasz T.: $B-594$

Glatzkova M.A.: SS MS 1

Gleeson F.: A-010, A-168

Gleeson F.V.: B-029, B-399

Glikstein R.: B-292

Glitsch U.: B-786

Glutig K.: $B-480$

Gnannt R.: B-382

Goerg F.: B-128

Gogbashian A.: B-803

Goh A.S.W.: B-151, B-152

Goh V.: B-803

Goh V.J.: A-096, A-136, B-729

Golan T.: $B-672$

Goldberg N.: B-290

Goldenberg R.: B-238

Goldstone A.: B-194

Gollwitzer H.: B-376

Gomes da Silva M.A.: A-231

Gomes R.: B-583

Gómez-Ansón B.: A-480, A-500

Gong J.: B-508

Gonzalez Graniel K.: B-592

Gonzalez Mirelis J.: B-201

Goo J.M.: B-024

Gore R.M.: A-334

Gori C.: B-865

Gorna R.: B-645

Götti R.: B-382

Gounis M.: B-502

Gourtsoyianni S.: B-256

Grabellus F.: B-051

Gradinger R.: B-091

Graessner J.: B-206, B-600

Graf R.: B-385

Grainger A.J.: A-201

Grand S.: B-511

Grande-Nagel I.: B-807

Grandin L.: B-237

Grant P.E.: $B-478$

Grant T.: $B-808$

Graser A.: A-410, B-323, B-711, B-794

Grasruck M.: $B-582$

Grassi R.: B-285, B-654, B-655

Green S.M.: $B-787$

Greenwood R.: B-433

Greiser A.: B-097

Gremizzi C.: B-386

Gremse F:: B-774

Grenacher L.: B-257, B-260

Grenet D.: B-651

Grenier N.: A-393, B-540, B-670

Greschus S.: $B-451$

Grgic A.: B-393

Grigoryev M.: B-078

Grimm J.: B-243, B-659, B-771, B-856 
Grimm J.M.: B-464

Grimmer R.: B-366

Grinias E.: B-648

Grobgeld D.: B-672

Groenewoud J.H.: B-806

Grommes J.: B-636

Gröschel T.: B-550, B-644

Grosse R.: B-206

Grosskurth D.: B-267

Grøvik E.: B-542

Gruber H.: B-287, B-717

Gruber-Rouh T.: B-032, B-033, B-067

Grünberg K.M.: B-135

Gryspeerdt S.: B-524

Gschwend S.: B-223

Gu S.: B-763

Guaraldi G.: B-615, B-617

Guaricci A.: B-236

Gueret P.: B-231

Guerini $\mathrm{H} .: B-568$

Guermazi A.: B-171, B-174, B-379, B-787

Guerra L.: B-021, B-119

Guggenberger R.: $B-570$

Guglielmi G.: A-322, B-261, B-262

Gui B.: B-063

Guidi L.: B-439

Guimarães L.S.: $A-135$

Guirguis R.: $B-741$

Guiu B.: B-750, B-764

Gujral G.S.: $B-106$

Gulay H.: B-079

Gullien R.: B-186, B-446, B-725

Gumustas S.: $B-515$

Günhan-Bilgen I.: A-047

Guo Q.Y.: B-259

Guo W: : B-761

Guo Y.: $B-347$

Gupta R.: B-254

Gupta S.: B-397

Guralnik L.: B-398

Gurung J.: B-035, B-423

Guruprasadh C.: $B-827$

Gutberlet M.: A-052, B-537

Guthrie A.: $B-426$

Guthrie J.A.: A-456

Gwinner W.: B-537

Györke T.: $B-738$

\section{H}

Haage P.: A-308

Haakenaasen U.: B-186

Haakull A.E.: B-446, B-725

Haan J.: $B-351, B-352$

Haberkorn U.: B-706

Haberland U.: B-102

Habermann C.R.: A-222

Habets J.: $B-824$

Habrecht H.: $B-550$

Hachulla A.-L.: A-382, B-752, B-756

Hadizadeh D.R.: $B-451$

Hadjiev J.: B-579

Haeck J.C.: B-560

Hafeez R.: B-522

Hagiwara A.: $B-364$

Hagspiel K.D.: $B-767$

Hahn D.: B-656

Hahn G.: B-480

Hahn S.Y.: B-674

Hainfellner J.: $B-215$

Haioun C.: A-095

Hájek M.: A-344

Halder J.: $B-680$
Halim M.Y.Z.: B-012

Halligan S.: A-072, A-076, A-172, B-329, B-330, $B-436, B-522$

Hallscheidt P.: A-394

Halter R.: $B-778$

Hamm B.: A-137, B-509, B-510, B-702

Hammerstingl R.: B-036, D-19

Hammon M.: B-124

Hammond R.L.: B-446

Hampshire T.: $B-329$

Hamza H.: B-605

Hamza H.M.H.: B-219

Han D.: $B-610, B-713$

Han D.H.: $B-275$

Han J.K.: B-015, B-168, B-251, B-611, B-842, B-849

Han M.R.: B-457

Han S.W.: $B-251$

Han Y.: $B-297, B-360$

Han Z.: $B-763$

Hanaoka S.: B-868

Haneder S.: B-052, B-342, B-532, B-607

Hänggi D.: $B-248$

Hannon M.J.: $B-787$

Hansch A.: $B-221$

Hansell D.M.: A-473, B-271, B-272

Hany T.F.: $A-013$

Hao D.P: $B-783$

Hao N.: $B-437, B-438$

Haraldseth $0 .: A-443$

Harder M.: $B-719$

Harrieder A.: $B-863$

Hartmann C.: B-214

Hartmann I.J.C.: B-592

Hartung B.: B-656

Hartung D.: B-537, B-778

Hartwig T.: $B-509$

Hasegawa K.: B-002

Hassepass F.: B-088

Hata S.: B-002

Hatakenaka M.: $B-418$

Hatlinghus S.: A-443

Hauenstein C.: $B-688$

Hauer R.N.W.: $B-830$

Haug A.: B-841

Haug U.: A-139

Hauger O.: A-202

Hausegger K.A.: A-120

Hawkes D.: B-329

Hayashi N.: B-868

Hayirlioglu A.: B-744

He B.: B-610

He V.: $B-199$

He W.: B-142

He Y.: B-495

Headden G.F.: B-492

Healey P.: B-447

Hebda A.: B-104

Hecher K.: $B-600$

Heerschap A.: A-343

Hegenscheid K.: $B$-131, $B-383$

Heiland S.: B-214

Hein P.: $B-821$

Heindel W.: B-182, B-294, B-295, B-647, B-779

Heine O.: B-702

Heinrichs E.-L.: $B-785$, $B-788$

Heintz A.: $B-530$

Heinz-Peer G.: A-115

Hekimoglu B.: $B-819$

Hekmatnia A.: B-020

Hekmatnia F.: $B-020$

Helbich T.H.: A-267

Helck A.: B-684

Helck A.D.: B-534

Hélénon O.: B-814
Hellerhof K.: B-074

Hellström P.: B-735

Hellwig D.: $B-393$

Helm E.J.: B-029

Hempel R.: B-716

Henes F.O.: $B-620$

Henner A.: A-331, B-577, B-580

Hennig J.: $A-104, B-598$

Hentrich D.: $B-125$

Hentschel H.: B-057

Henzler T.: B-023, B-334, B-342, B-412, B-413, $B-597, B-857$

Heredia V.: $B-539, B-619$

Hermans R.: A-529

Hernigou A.: B-395

Herold C.J.: A-333, A-527

Herrmann K.: $B-100, B-419, B-841$

Herrmann K.A.: B-225, B-681, B-848

Hertel A.: A-007

Herth F.: $B-278$

Herynek V.: $A-344$

Hes O.: B-793

Hess A.: B-371, B-456

Hesselmann V.: $B-294$

Hesterman J.: B-776

Heusner T.: B-144

Heusner T.A.: $B-118$

Heussel C.: $B-278$

Heussel C.P.: A-367

Heußel C.-P.: B-626

Heverhagen J.T.: $B-671$

Heye S.: B-345

Heye T.: $B-257, B-260$

Heys S.D.: $B-183$

Hierholzer J.: B-502

Hill C.: $B-400$

Hillman B.J.: A-359

Himmelreich U.: B-101

Hinkel R.: B-695, B-780

Hintze C.: $B-148$

Hirschenbein A.: B-858

Hiwatashi A.: B-107

Hlavacek A.: B-597

Ho Y.W.: B-852

Hoa D.: $B-281$

Hodler J.: A-029, B-385, B-536, B-570

Hoeffel C.: A-504

Hoehne-Hückstädt U.: $B-786$

Hoetker A.: B-530

Hoette S.: $B-420$

Hof J.R.: B-087

Hoffelner T.: B-789

Höffer J.: $B-267$

Hoffmann C.: A-276

Hoffmann M.H.K.: A-179

Hoffmann R.T.: $B-160$

Hoffmann U.: B-232

Hoffmann V.: B-232

Hoffstetter P.: B-085

Hofman P.A.M.: B-087

Hofmann B.M.: B-449

Hofmann W.: B-402

Hofstra L.: A-174

Hogan M.: B-292

Hohl C.: A-308

Holemans J.: B-708

Holland J.P.: $B-771$

Hollmann S.: B-208

Holmberg S.R.M.: B-332

Holmes K.G.: $A-444$

Holzapfel K.: B-305, B-376, B-421, B-484

Holzschuh M.: $B-257$

Hompes P.G.A.: B-529, B-795

Honda $\mathrm{H}$ : : $B-107, B-418$ 
Hong S.J.: $B-569$

Hoo Y.L.: B-045, B-633

Hoornaert M.-T.: $B-726$

Hoppe H.: A-532

Hoppin J.: $B-776$

Hora M.: $B-793$

Horng A.: B-176, B-567, B-786

Horsthuis K.: $B-392$

Hoshi T.: B-273

Hosoda K.: B-273

Hosokawa T.: $B-273$

Hosten N.: B-037, B-131, B-224, B-383

Hotz B.: $B-430$

Hotz H.G.: B-430

Hou Y.: B-259

Houterman S.: B-524

Houzard C.: $B-116$

Howarth N.: A-027, A-218

Hricak H.: A-137, A-355, B-302, B-665

Hrkac-Pustahija A.: B-077

Hu M.: B-329

Hu X.: B-336, B-859

Hu Y.-S.: $B-775$

Hua $Y .: B-370$

Huan Y.: B-545

Huang M.P.: $B-046$

Huber A.: B-210, B-234, B-291, B-348, B-552, $B-861$

Huber A.M.: B-100, B-419

Hubrecht R.: B-670

Huda W.: B-597

Huellner M.W.: $B-422$

Hueper K.: B-537, B-778

Huetter J.: B-704, B-710

Hughes M.: B-447

Hughes N.M.: SS MS 2

Hultcrantz R.: A-138

Humanes L.: $B-813$

Humbert M.: B-420

Hummel V.: B-059

Humphries P.: B-739

Humphries P.D.: A-453

Hunold P.: B-208, $B-807$

Hunter C.J.: B-526

Hunter D.J.: B-379

Hur C.: $B-459$

Hurdman J.: B-400

Husarik D.B.: $B-855$

Hustinx R.: A-009

Hüttl K.: B-635

Huysse W.: $B-785, B-788$

Huzjan-Korunic R.: B-077

Hwang D.: B-459

Hwang H.G.: B-822

Hwang H.Y.: $B-836$

Hwang K.W.: B-547

I

Ibolyka D.: B-594

Ichikawa T.: B-138

Idée J.-M.: B-226, $B-227$

lerace T: $B-290$

lezzi R.: $A-226, B-048, B-685$

lima M.: $B-543$

Illuminati S.: B-732, B-734

Im S.-A.: B-251

Inoue K.: $B-776$

Inoue M.: B-273

Invernizzi F.: $B-162$

lori M.: $B-589$

Ippolito D.: B-021, B-055, B-056, B-119, B-162, $B-252, B-253$
Irani F.G.: B-152

Irion $\mathrm{K}: \mathrm{B}-708$

Ishiguchi T.: $B-804$

Issever A.S.: $B-180$

Ith M.: $B-538$

Itti $\mathrm{E}:$ : A-095

Itzchak Y.: B-672

lussich $G$.: B-327

Ivanac $G$.: B-077

Ivanovas V.: B-073

$\overline{\mathbf{J}}$

Jacks L.M.: B-320, B-866

Jackson A.: A-520

Jackson J.E.: A-063

Jacob A.L.: B-341

Jacobi L.A.: $B-740$

Jacobi V.: $B-423, B-755, B-759$

Jacquier A.: B-417

Jaeger S.: $B-406$

Jaffe T.A.: $B-855$

Jaffer O.S.: B-612

Jahr G.: B-186

Jais J.P.: $B-814$

Jakicic J.M.: $B-787$

Jakobs T.F.: $B-160$

Jakobsen J.A.: $D-38$

Jambor I.: B-306

James S.: $A-516$

Jamieson F.: B-629

Jang H.C.: $B-499$

Jang Y.-J.: B-846

Janka G.E.: B-206

Jansen F.H.: B-805, B-806

Jansen P.L.M.: B-384

Janssen I.M.C.: B-384

Jaroni J.: B-419

Jarvinen $\mathrm{H}$.: $A-402$

Jaschke W.: B-554, B-662

Jayawardena M.: $B-708$

Jeantroux J.: B-568

Jégouic $C$.: $B-730$

Jehl F.: $B-606$

Jellus V:: $B-177$

Jenrette, III J.M.: B-218

Jeon C.H.: B-499

Jeon E.-Y.: B-410

Jerowski C.: $B-549$

Jia Q.X.: B-545

Jiang L.: $B-840$

Jiang S.: B-098, B-691

Jiang X.X.: B-164

Jin G.Y.: $B-630$

Jin K.N.: B-499

Jin L.: B-495

Jin P.: B-505

Jinnouchi M.: B-418

Jinzaki M.: $B-273$

Jirák D.: $A-344$

Jo Y.-I.: $B-639$

Johansson L.: B-559

Johnson J.-O.F.: B-397

Johnson L.: B-852

Johnson P.: A-110

Johnson T.: $B-411, B-711$

Johnson T.R.C.: A-003, A-018, B-391, B-758, B-794

Johnsrud K.: B-473

Johnsson Å.A.: $D-14$

Jones P.: $B-071$

Jones S.: B-071

Joo I.: B-015
José R.: $B-786$

Josseaume T.: $B-054$

Jost A.: B-289, SS MS 4

Jost G.: $B-704, B-710$

Jouannic J.-M.: B-835

Juchems M.S.: B-022

Jung H.J.: B-674

Jung J.I.: $B-275$

Jung M.: $B-440$

Junginger T: $B-530$

Junker D.: B-662

Junqueira F.P.: $B-692$

Junqueira V.C.N.: B-669

Juras V.: $B-178$

Jürgens M.: $B-729$

$\mathbf{K}$

Kachelrieß M.: B-363, B-365, B-366, B-367, $B-369, B-584$

Kahlert P.: $B-694$

Kahn C.E.: A-243

Kahn T.: B-007, B-149

Kainberger F.: A-022, B-127, B-179, B-604, B-745

Kaiser G.M.: B-694

Kaiser W.A.: A-158, A-433, B-221, B-318, B-319,

$B-487, B-549, B-550, B-642, B-643, B-644$,

$B-646, B-679, B-680, D-04$

Kaleda V.G.: $B-838$

Kalender W.: B-593

Kalender W.A.: A-134, A-215

Kamal K.: B-219

Kamano H.: B-107

Kamitani T.: $B-418$

Kamusella P.: $B-409$

Kanao S.: $B-543$

Kandel S.: B-258, B-331

Kang C.H.: B-569

Kang D.K.: B-334

Kang M.: B-254

Kapsimalakou S.: B-208, B-807

Karaguzel G.: B-768

Karami Nogourani M.: B-082

Karantanas A.H.: A-194, A-263, B-782, B-784

Karcaaltincaba M.: A-266

Karcz W.K.: B-018

Karim S.: $B-867$

Karlinger K.: B-594

Karssemeijer N.: B-181

Karunasaagarar K.: $B-400$

Kashani H.: B-339

Kaskati T.: $B-079$

Kasmai B.: B-433, B-434

Kasprian G.: B-215, B-471, B-472, B-800, B-832, $B-833, B-834$

Kassarjian A.: A-461

Kastler A.: B-762

Kataoka M.: B-283

Kato S.: B-092

Katoh M.: A-033, B-406, B-632, B-690

Kauczor H.U.: B-257, B-260

Kauczor H.-U.: A-447, A-475, B-045, B-110, $B-278, B-587, B-626, B-633, B-706, B-867$

Kautzky-Willer A.: $B-790$

Kavanagh L.: B-469

Kawabata Y.: B-273

Kawaguchi H.: B-476

Kawai K.: B-255

Kayed H.: B-112, B-199

Kbilashvili N.: B-212

Keck J.: $B-375$

Keeling A.: A-373

Keignart S.: B-511, B-512 
Kelekis A.: B-503, B-506

Kelekis A.D.: A-146

Kelekis N.: B-503

Kelekis N.L.: B-506

Kellenberger C.J.: A-131

Keller J.: A-521

Kelly B.: $B-444$

Kemerink G.J.: B-740

Kemmling A.: B-294, B-295

Kemppainen J.: $B-306$

Kenn W.: B-656

Kennish S.J.: $B-664$

Kerl J.M.: B-193, B-697, B-753, B-755, B-759

Kerl M.J.: B-445

Kerner-Bignell A.: B-434

Kessler J.M.: B-586

Kettenbach J.: A-419

Keulers A.: B-749

Khandelwal N.: B-254, B-827

Kheddache S.: $D-14$

Khedr S.: B-053, B-561, B-562

Khoo L.S.S.: $B-152$

Kichikawa K.: B-133

Kickhefel A.: B-383

Kido K.: $B-581$

Kiely D.: $B-400$

Kienle K.-P.: $B-375$

Kiessling F.: $B-774$

Kiessling F.M.A.: A-173, A-468

Kim B.H.: B-569

Kim C.K.: B-062, B-667

Kim D.: B-251

Kim D.H.: B-490

Kim E.: B-090

Kim E.J.: B-457

Kim G.-C.: B-846

Kim G.H.: B-490

Kim H.J.: B-410

Kim H.-J.: B-090

Kim H.S.: B-062, B-674

Kim I.: B-459

Kim J.H.: B-024, B-836

Kim K.S.: B-459

Kim K.W.: B-849

Kim M.-J.: B-004

Kim S.: $B-490$

Kim S.H.: B-015, B-168, B-251, B-611

Kim S.K.: B-630

Kim S.T.: B-090

Kim S.Y.: B-457

Kim T.H.: B-457

Kim T.Y.: $B-569$

Kim Y.G.: B-269

Kim Y.J.: B-275, B-822

Kim Y.-J.: B-375, B-379

Kim Y.L.: B-569

Kimmig R.: B-118

Kimpe T.: $B-865$

Kimura J.: $B-804$

King A.D.: A-530

Kinner S.: B-486, B-535

Kinsella C.: $B-576$

Kinsella S.: $B-727$

Kiritsi O.: $B-466$

Kirsch C.-M.: B-393

Kitagawa K.: B-092

Kitajima K.: B-820

Klammsteiner N.: B-554

Klass O.: B-022

Klauser A.: A-311, D-24

Klauß M.: $B-135$

Klausz R.: B-724

Klein I.: B-093

Kleinman P.K.: A-036, A-153
Kleinrensink G.J.: B-175

Kleven-Madsen N.: B-442

Klijn S.: B-307

Klima M.: B-190

Klinge U.: $B-653$

Kloska S.: B-455

Klotz E.: B-102, B-293, B-359, B-729

Klutmann S.: $B-058, B-467$

Knauth M.: B-102, B-105, B-359

Ko S.M.: $B-822$

Ko Y.: B-459

Kobayashi S.: $B-255$

Kober F.: $B-417$

Koch A.M.: B-592

Kochhar R.: B-019

Koda W.: B-255

Koehli M.: B-428

Koeninger A.: $B-118$

Koga S.: $B-273$

Koh D.-M.: A-273, D-16, D-21

Kohlhase N.: B-647

Kohli A.: $B-616$

Kokudo N.: B-002

Kool D.R.: $A-424$

Kopp I.: $B-600$

Koral K.: B-837

Körner M.: B-591, B-716, B-856, B-863

Kosanke Y.: $B-113$

Koscielny S.: $B-425$

Kott A.: B-190, B-645

Kotter E.C.: B-073

Koutalonis M.: A-494

Kovacs A.: B-208, B-451, B-579, B-807

Koyama H.: $B-143$

Kozák L.R.: B-738

Kozaka K.: $B-255$

Kozawa E.: $B-358$

Kraemer N.A.: B-128

Kraff O.: $B-535$

Krainik A.: A-123, B-511, B-512

Kramer J.: A-082

Krämer N.A.: B-653

Kramer S.: $B-755$

Kramp L.: B-214

Krasnokutsky S.: B-176

Kratovac-Dunjic M.: B-195

Kraus T.: $B-088$

Krause B.J.: A-015

Krausé D.: $B-750, B-764$

Krauss B.: $B-609$

Kreft A : $B-530$

Kreitner K.-F.: A-486, B-041

Kremser C.: B-554

Krestan C.R.: $A-150$

Krestin G.P.: A-439, B-175, B-416, B-696, B-860

Kreuzberg B.: B-296, $B-793$

Krings T.: $A-524$

Krissak R.: B-857

Kŕ̛iž J.: A-344

Kröger N.: B-057, B-058

Krombach G.: $B-640$

Krombach G.A.: B-653

Krompiec C.: B-640

Kröpil P.: B-248

Krssak M.: B-215, B-745

Krumina G.: $A-413$

Kubiena H.: A-064

Kuchta J.: B-267

Kudrnova S.: $B-635$

Kuhl C.: $B-653, B-749$

Kuhl C.K.: A-269, A-389, B-128

Kühn J.P.: $B-224, B-383$

Kühn J.-P.: B-131

Kühnert N.: B-653
Kulemann V.: B-834

Kulkarni S.: B-313

Kumar R.M.: B-827

Kunhigk J.-M.: B-028

Kuribayashi S.: $B-273$

Kusamura S.: B-014

Küttner A.: A-108

Kuzmiak C.: B-548

Kwoh C.K.: B-787

Kwon H.: B-490

Kwong Y.: B-265, B-266

\section{L}

La Grassa M.: B-187

la Parra C.: $B-154$

Lacelli F.: B-081, B-501

Lackas C.: B-776

Lacroix J.: $B-425$

Ladd M.E.: B-535, B-694

Ladd S.: $B-144$

Laghi A.: A-074, A-140, A-318, B-416

Lai L.: B-245, B-357

Laissy J.-P.: B-093, B-209, B-231

Lakosi F.: B-579

Lam W.W.: B-852

Lamberto S.: B-346

Lambregts D.M.J.: B-161, B-163, B-165, B-167, $B-169, B-528$

Lammer J.: A-178, A-247, A-509

Lamy de la Chapelle T.: $B-054$

Lanca L.J.O.C.: A-329, B-441

Lanconelli N.: B-589

Lanczi L.I.: SS MS 4

Landi N.: B-654, B-655

Lang C.: $B-823$

Lange P.: $B-700, B-825$

Länger $F$.: $B-778$

Langer M.: B-018, B-026, B-073, B-088, B-598, $B-599, B-609, B-823$

Langer S.: $B-636$

Langs G.: $B-127, B-832$

Laniado M.: A-316

Lannegrand Menéndez B.: B-086

Lanzman R.S.: B-248, B-531

Lapeña L.: B-115

Laqmani A.: B-623

Larici A.R.: A-026, A-328, B-146, B-279

Lario C.: $B-498$

Laroche D.: B-701

Larralde A.: B-345

Larsen C.K.: B-660

Lascialfari A.: $B-590$

Lassau N.: $B-425, D-29$

Latief K.: B-265, B-266

Laudi C.: $B-327$

Lauenstein T.C.: B-051, B-118, B-486, B-535

Lauer L.: B-173

Launay-Vacher V.: $B-230$

Laurent A.: B-850

Laurent $\mathrm{F}$ : $A-366, B-228$

Lauria F.: $B-650$

Lavini C.: $B-435$

Law M.: A-163

Lawler L.P.: A-241

Lawrence G.: $B-183$

Lawson A.: B-371, B-733

Layré B.: $B-068$

Le Bas J.-F.: B-511, B-512

Le Bihan D.: A-071, B-543

Le Bot R.: B-227

Le Bras Y.: B-670

Le Corroller T.: B-565 
Le Guennec L.: B-456

Le Viet D.: B-568

Leahy M.: B-019

Leander P.: B-707

Lebedeva I.S.: $B-838$

Leber K.: $B-250$

Leclerc X.: A-124

Lecluyse C.: $B-017$

Lecouvet F.E.: A-275, B-563, B-564

Lederle W.: $B-774$

Lederman R.B.: B-188, B-189

Lee E.S.: B-547

Lee G.Y.: $B-569$

Lee H.: B-090

Lee H.J.: B-024, B-639

Lee I.: $B-836$

Lee J.M.: B-015, B-168, B-842, B-849

Lee J.-M.: B-846

Lee J.S.: $B-457$

Lee J.W.: B-269

Lee J.Y.: $B-251, B-611$

Lee K.S.: $B-836$

Lee M.: $B-469$

Lee M.J.: $A-213$

Lee N.K.: $B-490$

Lee R.-C.: B-683

Lee S.J.: $B-822$

Lee S.M.: B-024

Lee S.-M.: $B-846$

Lee Y.: $B-499$

Lee Y.H.: B-200

Leeuwenburgh M.M.N.: B-652

Lefere P.: A-073, B-524

Lefevre S.: $B-606$

Lefort C.: $B-395$

Lehner F.: $B-537$

Lehnert T.: B-032, B-033, B-034, B-067, B-445, $B-697, B-755$

Leichter I.: $B-188, B-189$

Leifland K.: B-185

Leijtens J.W.A.: $B-167$

Leiner T.: B-344, B-350

Lell M.: $B-365, D-11$

Lell M.M.: B-293, B-593

Lely R.J.: $B-392$

Lembcke A.: $B-331, B-821$

Lemke A.: $B-135$

Lemke H.U.: A-538

Lemuhot M.: $B-724$

Lencioni R.: $A-513$

Lengsfeld P.: B-704, B-710

Lenhard D.: B-704, B-710

Leni D.: $B-252, B-253$

Leonardi M.: A-296

Lepanto L.: B-769

Lepomäki V.: B-306

Lerais J.-M.: B-750

Lerch M.: B-131

Leschka S.: A-053, A-427, B-382, B-854

Lesnik Oberstein S.A.J.: B-351, B-352

Leuchte H.: B-391

Levy M.: $B-555$

Lewis C.E.: B-171, B-174

Lewis J.S.: $B-771$

Ley S.: B-045, B-278, B-626, B-706

Leyvraz S.: B-428

Ley-Zaporozhan J.: B-278

Li D.: B-495

Li H.: B-557

Li J.: B-010, B-142, B-347, B-370, B-798

Li J.-Y.: $B-816$

Li K.: $B-299$

Li L.: $B-379, B-505$

Li M.: B-249, B-453, B-505, B-714
Li N.: $B-042$

Li S.: $B-803$

Li T.: $B-360$

Li W.: $B-618$

Li X.-T.: $B-816$

Li Y.: B-030, B-297, B-453, B-470

Li Y.-J.: $B-775$

Li Z.: $B-008$

Li Z.-P.: B-557

Liang C.: $B-387$

Liang C.H.: B-046

Liapi E.: $B-156$

Liardo E.V.: $B-117$

Liebig T.: $B-291$

Liem M.K.: B-351, B-352

Ligabue G.: B-117, B-202, B-615, B-617

Lilic G.: B-195

Lim H.J.: B-547

Lim J.H.: B-792

Lim S.: $B-499$

Lim Y.C.: $B-457$

Lima Passos V.: B-184

Lima T.: $B-708$

Lin C.: A-095

Lin M.D.: B-156

Lin W.: $B-859$

Lin X.: $B-010$

Linda A.: B-072, B-311, B-312, B-315

Lindemayr S.: $B-036$

Link T.M.: $B-172, B-180, B-263, B-264$

Linn J.: B-355, B-588

Linsenmaier U.: B-591, B-716, B-856, B-863

Lisbona M.P.P.: $B-602$

Lisbona R.: $B-526$

Lisi D.: B-301, B-666

Littvay L.: $B-241$

Liu C.: $B-042$

Liu H.: B-387

Liu J.H.: $B-783$

Liu P.-F.: B-429

Liu Q.: B-691

Liu S.: B-065, B-691

Liu S.F.: $B-852$

Liu S.-Y.: $B-141$

Liu T.: $B-558$

Liu X.: B-304

Liu Y.: $B-170, B-839$

Liu Z: $B-145$

Lloyd D.: $B-748$

Lo G.: B-852

Lo R.: $B-151$

Lo R.H.G.: B-152

Lo Zupone C.: B-732, B-734

Lobbes M.: B-184

Lobo M.L.: A-497

Lobsien D.: B-007

Lodder W.L.: B-191

Lodemann K.-P.: B-604

Loewe C.: A-440, B-345

Loffroy R.: $B-156, B-750, B-764$

Loizides A.: B-287, $B-717$

Lombardi M.: B-207

Lombardo A.: B-099

Londero V.: B-072, B-311, B-312, B-315

Lord B.: $B-286, B-320, B-866$

Lord M.: $B-772$

Loreni G.: B-404

Lorenzen M.: B-620

Lorenzon M.: B-072, B-312

Loria P.: $B-615, B-617$

Lorusso A.: B-001

Losio C.: B-014, B-386, B-673

Lotersztajn S.: $B-555$

Loubeyre P.: B-772
Louwman M.W.J.: B-806

Lovato L.: B-203

Lovrenski J.: B-595

Löwik C.W.G.M.: A-469

Lu G.: B-454, B-754, B-760

Lu J.: $B-299$

Lu L.: B-454, B-754, B-760

Lu M.: B-098, B-691

Lu T.-L.C.: $B-427$

Luchinger A.B.: $B-795$

Luciani A.: A-095, B-555, B-850

Luciani M.: B-546, B-676, B-678

Lucidarme O.: A-206

Luijkx T.: B-340, B-830

Lummel N.: $B-588$

Lung P.: $B-612$

Luo B.N.: B-840

Luo S.: $B-754$

Luo Z.: $B-715$

Luster M.: B-022

Luzietti M.: B-202

Lv P.: B-010

Lynch J.: B-172

Lysdahl K.B.: B-449

\section{M}

Ma D.: B-142

Ma N.: B-098

Maas M.: A-145, B-161, B-163, B-165, B-167, $B-169, B-528$

Macchi I.: B-056

Macchia G.: B-064

Maccioni F.: A-171

Machin D.: $B-151$

Mack M.G.: A-286, B-031, B-193, B-289

MacLennan G.S.: $B-183$

Mader C.: B-854

Maderwald S.: $B-535$

Madureira A.J.B.S.: A-106

Maeda T.: B-820

Maentele W.: $B-147$

Maffei E.: A-425, B-236, B-498

Maffi P.: $B-386, B-462$

Maggini $E .: B-815$

Magnaldi S.: $B-844$

Mahalingam S.: $B-132$

Mahgerefteh S.Y.: $B-858$

Maher M.M: B-718, B-727, D-40

Mahnken A.H.: B-636, B-749, D-10

Maier A.: A-477

Maiorano E.: B-192

Maiuri F.: $B-479$

Maiwald B.: B-007

Majos C.: B-217

Majumdar S.: B-180

Makris A.: B-803

Maksimovic R.: B-195

Malagari A.: B-506

Malagari K.: $A-511$

Malago R.: $B-333, B-496$

Malavaud B.: $B-303$

Malchair F.: $B-726$

Malcolm P.: B-433, B-434

Maldur V.: $B-388$

Mali W.: B-071

Mali W.P.T.M.: B-340, B-824, B-830

Malich A.: B-125, B-190, B-645

Malinen E.: $B-473$

Mall P.: $B-430$

Mallarajapatna G.J.: B-284

Mallarini G.: B-242, B-244, B-245, B-357, B-504

Mallett S.: $B-330$ 
Malone D.E.: A-249, A-315

Maly Sundgren P.C.: $A-164$

Mamisch T.C.: A-256, B-173, B-178, B-375, B-378, $B-379, B-601, B-790, D-34$

Mancuso E.: $B-815$

Mancuso F.: B-204

Mandolfino T:: $B-346$

Manegold C.: B-023

Manelfe J.: B-068

Manfredi A.: $B-781$

Manfredi R.: B-134, B-797

Mang T.: A-409

Manganaro L.: B-693

Mangini M.: B-631, B-634

Manichon A.F.: B-116

Manin S.: B-555

Manning D.: $B-330$

Månsson L.G.: $D-14$

Mantarro A.: B-326

Mantini D.: B-520

Maqbool M.: SS MS 3

Mara M.: B-066

Marangoni R.: $B-345$

Marano R.: B-094, B-095

Marcantonio A.: B-388

Marchesi V.: $B-117$

Marchianò A.: B-028

Marcia S.: $B-504$

Marcus R.: $B-411, B-758$

Marigliano C.: $B-388$

Marin D.: $B-855$

Marini M.: $B-693$

Marini S.: $B-504$

Maris T.G.: $A-407$

Markl M.: B-598, B-599, B-688

Markovic D.: B-570

Marlovits S.: $B-173, B-790$

Maroldi R.: A-528, A-531, B-196

Marra M.D.: B-171, B-174

Marras M.: B-504

Marras T.: $B-629$

Marti M.: B-621, B-622

Martin Boizas R.: B-086

Martín C.: A-237

Martin J.: B-194

Martincich L.: B-671

Martínez C.: B-233

Martínez Rubio C.: B-317

Martini C.: B-236, B-372, B-373, B-498

Martinoli C.: $A-310, B-479$

Marugami N.: $B-133$

Mas J.-L.: B-456

Masala S.: A-465

Masciocchi C.: $A-378$

Massari F.: $B-460$

Masselli G.: A-089

Massmann A.: B-632, B-690, B-699

Mastantuono M.: B-507

Masutani Y.: B-868

Mathies R.: B-402

Matos C.: A-364

Matsui O.: $B-255$

Matsumoto A.H.: B-767

Matsumoto K.: $B-143$

Matsumoto S.: B-143

Matsuo Y.: B-418

Matthews K.: B-578

Matthews S.: $B-027$

Matzek W.: B-129

Maubon A.: B-059

Maubon A.J.M.: B-068

Mauch J.: B-599

Mauerer A.: B-173

Maurel B.: B-565
Maurer M.H.: B-702

Mauri G.: B-047

Maurício J.C.: B-441

May M.: B-593

Mayer M.: B-789

Mayerhoefer M.E.: B-178

Mayerhöfer M.E.: B-129

Maymo J.G.: B-602

Mazaheri Y.: B-302

Mazioti A.: B-503, B-506

Mazonakis M.: $B-648$

Mazzei F.G.: $B-650$

Mazzei M.A.: B-650

Mazziotti S.: B-791

Mazzoni L.N.: B-482, B-483, B-485

Mbalisike E.: $B-158$

Mbalisike E.C.: B-034, B-035, B-423, B-424

Mc Entee M.F.: B-444, B-576

Mc Garrigle A.M.: B-718

Mc Laughlin P.: B-718

McCann C.A.: B-708

McCarthy C.J.: B-870

McCarthy S.: B-727

McClelland J.: B-329

McCulloch C.E.: B-172

McDougall I.: B-694

McEntee M.: B-578

McEntee M.F.: B-571, B-573

McKillop G.: B-338

McWilliams E.T.M.: B-332

McWilliams S.: B-727

Medda E.: B-241

Méder J.-F.: $B-456$

Meduri A.: B-094, B-095, B-099

Medvedeieva O.: B-815

Mehrez H.: B-337

Mehta D.: B-322

Meier R.: B-113, B-291, B-603

Meier-Meitinger M.: $B-802$

Meijer J.: B-384

Meijer R.C.A.: B-824

Meijer T.S.: $B-824$

Meindl T.: A-196

Meintjies E.: $B$-371

Melikoglu M.: B-768

Meloni F.: B-253

Memin A.: B-511

Mendelson R.M.: A-080

Meneghetti G.: B-241

Menezes R.: B-337

Meng Q.F.: B-060

Meng Q.-F.: B-557

Menig M.: B-530

Mennini M.L.: B-005, B-624

Menovsky T.: $B-458$

Mensel B.: B-037

Mentore K.: B-531

Mentzel H.-J.: B-221

Menu Y.: A-138, A-399

Mercadal S.: $B-217$

Merhemic Z.: A-437

Merkus D.: $B-696$

Meroni S.: B-025

Messa C.: B-021, B-119

Messina C.: SS MS 2

Messner A.: B-790

Mester J.: B-467

Metafratzi Z.M.: B-276

Metz S.: $B-421$

Metzger L.: $B-532$

Metzgeroth G.: B-052

Meuli R.: $B-428$

Meuli R.A.: A-519

Meunier F.: A-271
Mews J.: B-331

Meyer E.: B-363, B-365

Meyer G.: B-395

Meyer H.: B-258, B-331

Meyer M.: B-334, $B-468$

Mezzanzanica M.: B-525

Mezzetta L.: B-764

Miao F.: B-437

Micchìa M.G.: B-493

Miccò M.: B-063

Michaely H.J.: B-052, B-342, B-532, B-607

Middleton S.J.: B-012

Midiri M.: $B-103$

Midulla M.: $B-043$

Miese F.R.: $B-248, B-601$

Miglio E.: B-544, B-546, B-676, B-678

Mijatovic V.: B-529, $B-795$

Miki S.: $B-868$

Mlkroulis G.: $B-466$

Mikulik S.: B-125

Mikuz G.: B-662

Milenkovic R.: B-195

Miles K.: B-013, B-332

Miles K.A.: A-136

Mileto A.: B-791

Millar F.R.: $B-338$

Mills P.: $B-071$

Mills T.: $B-120$

Milosevic Z.C.: B-810

Milovanovic J.: B-195

Miminoshvili D.: $B-354$

Minami T.: $B-255$

Minar E.: A-225

Minko P.: B-406

Minn H.: B-306

Minnerup J.: B-294, B-295

Minniti G.: $B-108$

Miodek F.: $B-393$

Mírka H.: A-155, A-426, B-296, B-793

Mishah N.M.: $B-448$

Missere M.: B-064

Mistretta C.A.: B-857

Mitter C.: B-472, B-833, B-834

Miyagi $\mathrm{H} .:$ B-092

Miyake $\mathrm{H} .: \mathrm{B}-820$

Mizukoshi W.: B-358

Moa E.: $B-185$

Modo M.: A-342

Möhlenbruch M.: B-451

Mohr M.: B-037

Molin D.: B-559

Molinari C.: $B-315$

Molinari F.: B-130

Molinaro S.: B-207

Molisso L.: B-049, B-349, B-491, B-507, B-766

Mollet N.R.: B-416, B-696, $B-860$

Moltz J.H.: B-649

Momose A.: B-581

Monaghan C.: B-708

Monetti F.: B-677

Monfardini L.: $B-282$

Monguzzi L.: B-119, B-162

Monina M.: B-353

Monpeyssen H.: B-814

Montalvan B.: B-568

Montani D.: B-420

Montani M.: B-385

Montefrancesco, R.: B-009

Monteil J.: B-059

Monteiro M.: B-572

Montemurro M.: B-428

Monti C.: $B-150$

Monti L.: B-732, B-734

Montisci R.: B-242, B-244, B-245, B-357 
Moog P.: B-603

Moon S.K.: B-015, B-611

Moore C.: $B-787$

Moore R.J.: $B-447$

Morana G.: A-188, B-137, B-624, B-625

Morcos S.K.: A-113

Moreaud O.: $B-511, B-512$

Moreira N.C.: $B-831$

Morella M.: B-192

Morello R.: $B-684$

Moreno R.: B-043, B-414

Morgan R.: A-119

Morhard D.: $B-711$

Morin J.: $B-769$

Morisaka H.: B-138

Moriyama N.: $B-776$

Mortilla M.: B-731

Moschetta M.: B-192

Moskowitz C.: B-302

Mostbeck G.H.: A-369

Motosugi U.: $B-138$

Mousavi M.: B-179

Moutard M.-L.: B-835

Mouton C.: B-701

Mpouhra K.: B-390

Mubashar M.: B-743

Mucci A.: B-615

Mück F.: $B-856$

Muda A.: B-742

Mueller D.: $B-180$

Mueller H.: B-853

Mueller S.P.: A-006

Mueller-Eschner M.: B-867

Muenzel D.: B-234, B-291

Muglia V.F.: $B-669$

Muin D.: B-129, B-844

Mull M.: A-524

Mullan D.P.G.: $B-447$

Müller A.: B-699

Müller D.: B-263

Müller P.: B-567

Muller R.: B-637

Muller S.: B-724

Müller-Eschner M.: B-045, B-633

Munkeby B.H.: B-473

Muntane A.: B-217

Münter M.: $B-135$

Murchison J.T.: $B-394$

Murolo C.: B-288, B-432

Murphy-Lavallée J.: B-769

Muscogiuri G.: B-204

Musset D.: $B-420, D-22$

Mussmann B.R.: A-283

Mut M.: B-211

Muzzio P.C.: B-817

Myhre H.O.: A-443

Mýrdal G.: A-396

\section{N}

Nadrljanski M.: B-810

Nagao M.: $B-418$

Nagashima M.: B-581

Nagata M.: B-092

Nägele T.: $B-216$

Naguib N.: $B-158, B-445$

Naguib N.N.: B-031, B-032, B-033, B-034, B-159, $B-289, B-737$

Naguib N.N.N.: B-067

Nakajima R.: B-358

Nakajima Y.: B-133

Nakano S.: B-804

Nance J.W.: B-334, B-468, B-492
Nance J.W.J.: $B-413$

Nandakumar A.: $B-746$

Nanz D.: B-385, B-536, B-570

Napoli A.: B-049, B-343, B-349, B-491, B-507, $B-766, D-36$

Napolitano C.: B-095

Näppi J.: $B-328$

Nardella V.G.: $B-828$

Nardi C.: B-482, B-483, B-485

Nardo L.: $B-172$

Nassif A.: $B-224$

Natale L.: A-485, B-094, B-095, B-099, B-202

Nauer C.: $B-575$

Navallas M.I.: B-602

Navarro M.: B-813

Nederend J.: $B-806$

Nederveen A.J.: B-384, B-435

Neefjes L.A.: $B-416$

Negård A.: $B-660$

Negre-Salvayre A.: $B-043$

Nelemans P.J.: B-344, B-350, B-528

Nelson R.C.: $B-855$

Nemec S.F.: B-800

Nemec U.: B-800

Nemes B.: B-635

Nemoto M.: $B-868$

Neri E.: A-07O, B-123, B-324, B-325, B-326, B-523

Nestola M.: B-048

Neubauer H.: B-656

Neumaier K.: $B-711$

Neuville A.: B-606

Neuwirth J.: A-190

Nevitt M.C.: B-171, B-172, B-174

Newby D.E.: $B-338$

Newton T.: B-337

Nguyn E.: B-337

Ni Y.: B-551

Niarra R.: $B-395$

Nicholson A.: A-031

Nicol E.D.: $B-335$

Nicolaides A.: B-130

Nicolau C.: A-014

Nicoud F.: $B-414$

Nieboer K.: B-851

Niederstadt T.: B-294, B-295

Nielsen P.: B-206

Niessen W.: B-556

Niessen W.J.: B-560

Nievelstein R.A.J.: A-406

Niggemann P.: B-267

Nijenhuis R.J.: A-524

Nikolaou K.: $A-533, B-232, B-243, B-391, B-411$, $B-464, B-553, B-684, B-695, B-700, B-758$, $B-780, B-825$

Nilsson I.-L.: B-111

Nilsson M.: B-707

Ning E.: $B-142$

Nishi N.: B-358

Nishio M.: $B-143$

Nishiofuku H.: B-133

Nitrosi A.: B-589

Niwa T.: $B-476$

Nocetti L.: B-615, B-617

Noebauer-Huhmann I.-M.: B-745

Noel P.: B-603

Noel P.B.: B-234, B-348, B-861

Nomura Y.: $B-868$

Noordhoek N.: B-156

Notohamiprodjo M.: B-534, B-567, B-681, B-684, B-786

Nour Eldin N.E.: B-289

Nour-Eldin M.: B-445

Nour-Eldin N.E.: B-031, B-034

Nour-Eldin N.-E.A.: B-032, B-033, B-067, B-737
Noussios G.: B-466

Novelline R.A.: B-397

Ntailiani A.: B-782, B-784

Ntoulia A.: B-736

Ntziachristos V.: $B-552$

Numanoglu A.: B-733

Nurmi M.: $B-306$

Nyman U.: B-707

Nyström N.: B-735

$\mathbf{0}$

O' Neill J.E.G.: B-441

O Neill S.: $B-718$

Obata T: : $B-476$

Oberhofer N.: B-722

Oberholzer K.: B-041, B-530

Obernauer J.: $B-287, B-717$

Oberoi S.: B-468, B-492, B-500, SS MS 3

Occhionero M.: B-064, B-381

Occhipinti M.E.: B-279

O'Connell M.: A-016, A-056

Odedra D.: B-339

Ødegård A.: $A-443$

Oei E.H.G.: $B-175$

Ogunwale B.: $B-743$

Oguz K.K.: B-211

Oh J.: B-275

Oh Y.W.: B-547

Ohno Y.: B-143, B-820

Ohshima Y.: B-804

Ohtomo K.: B-868

Okada T.: B-543

Okada Y.: B-358

O'Keeffe S.: B-080

Okujava M.: B-354

Okumura R.: $B-543$

Ola A.: B-032, B-033, B-737

O'Leary D.: B-574, B-808, B-809

Olerud H.: B-660

Olive M.: B-815

Olivié D.: B-769

Omidifar N.: B-020

Omoumi P.: B-563, B-564

Onishi Y.: $B-143$

Ooi L.L.P.J.: B-151

Opfermann K.J.: B-218

Oppenheim C.: B-456

Oreti D.S.: A-284

Origgi D.: $B-025$

Orlandi D.: B-372

Orliaguet I.: $B-116$

Orsenigo E.: B-014

Ortega A.: B-115

Ortiz L.: B-115

Orzada S.: $B-535$

Osawa A.: B-583

O'Shaughnessy E.: D-32

Ostendorf B.: B-601

Østerås B.H.: B-725

Ostermann S.: B-287, B-717

Oswald S.: B-224

Otani K.: B-255

Ott D.: B-538

Ott S.: B-455

Otterbach F.: B-118

Otto J.: B-653

Ou S.: B-239

Ouchchane L.: B-762

Oudkerk M.: B-280

Owen S.E.: $B-747$

Owens C.M.: A-129

Oyen W.J.: A-343

Ozgen Mocan B.: B-211 


$\overline{\mathbf{P}}$

Paalimäki-Paakki K.: A-285

Pablo L.: B-084

Pabst T.: $B-656$

Pace E.: B-489

Pache G.: B-026, B-088, B-609, B-823

Padhani A.R.: A-156, A-457

Padley S.P.: $B-335$

Padley S.P.G.: A-380

Padrenostro M.: $B-282$

Paeng J.C.: B-024

Pagniez J.: B-756

Pagonidis K.: $B-648$

Paldino M.J.: $B-478$

Palkó A.: A-299

Pall J.: $B-579$

Pallwein L.: B-662

Palmisano A.: $B-386, B-462$

Palmowski M.: A-470, $B-774$

Pameijer F.A.: A-087, B-191

Pampana E.: B-404, B-405, B-460

Pan H.H.: B-235

Pandolfi E.M.: B-663

Panebianco V.: B-301, B-308, B-666

Panicek D.M.: A-332

Panizza P.: $B-673$

Panotopoulos J.: $B-745$

Pans S.: A-517

Papa M.: B-672

Papadakis A.E.: B-321

Papadopoulou F.: B-736

Papakostas S.: $B$-298

Papanikolaou N.: A-363

Papathanasiou M.A.: A-482

Paprottka P.M.: B-160, B-553, B-780

Parent F.: $B-420$

Parentini B.: $B-153$

Pariente D.: A-126

Parizel P.M.: A-271, A-295, A-436, A-523, B-458, $B-513, B-517$

Park A.W.: B-767

Park B.K.: B-062, B-667

Park C.M.: B-024

Park E.K.: B-547

Park H.S.: B-842

Park J.-M.: B-410

Park K.H.: B-547

Park M.-S.: B-004

Park S.H.: B-275, B-328, B-674

Park S.W.: B-639

Park S.Y.: B-667

Park W.: B-667

Parker A.T.: $B-497$

Parlato V.: $B-285$

Parodi A.: B-432

Parohl N.: B-694

Parthipun A.: B-811

Partovi S.: B-341, B-633

Pasetto S.: B-590

Pasi N.: B-209

Pasin M.: B-481, B-675

Paso $\mathrm{H}$.: $B-577$

Pasquinelli F.: B-482, B-483, B-485

Passariello R.: B-005, B-049, B-277, B-301, $B-308, B-343, B-349, B-388, B-488, B-491$, $B-507, B-544, B-546, B-666, B-676, B-678$, $B-766, B-815$

Passera E.: $B-150$

Pasticier G.: B-670

Pastorelli D.: B-657

Pastorino U.: B-028

Pastrana Ledesma M.A.: B-201

Patak M.A.: A-305, B-751
Patel N.: B-198

Patel V.: $B-612$

Patrick C.: $A-221$

Patsch J.: B-127, B-179

Patsios D.: $B-629$

Pattacini P.: B-589

Patterson C.M.: B-335

Paul D.: B-173, B-177

Paul J.: B-147

Paul J.-F.: $B-237$

Paul N.: B-331, B-821

Paul N.S.: B-337, B-339

Pauls S.: $B-022$

Pauly V.: B-565

Pedersen M.: B-534

Pediconi F.: B-544, B-546, B-676, B-678

Pedrosa I.: $B-531$

Peer S.: B-287, B-717

Peitgen H.-O.: B-649

Pekar B.P.: B-744

Pekarovic D.: A-330

Peldschus K.: B-058

Pelegretti P.: B-073

Peng G.: $B-239$

Peng W.: B-304

Peng W.-J.: B-671

Peng Y.: B-839

Peng Z.-H.: B-249, B-505, B-714

Pepe A.: B-381

Perego P.: B-061

Peregrin J.H.: $A-210$

Pérez L.: $B-813$

Périat D.: $B-247$

Perisinakis K.: $B-321$

Perju-Dumbrava L.: B-472, B-834

Perotti C.: $B-703$

Perseghin G.: $B-205$

Persson A.: A-450

Pescarini L.: B-187

Peters J.F.: B-392

Petraikin A.V.: $B-838$

Petralia G.: B-009, B-481, B-675

Petrelli A.: B-462

Petrillo A.: B-671

Petrini M.: SS MS 4

Pezzato A.: B-333, B-496

Pezzetti G.: $B-781$

Pfefferkorn T.: $B-355$

Pfeil A.: B-221

Pfirrmann C.W.A.: A-262, B-377

Pfister D.: $B-128$

Pflugmacher R.: B-502

Pham C.T.: B-651

Philips N.R.: B-743

Phillips P.: B-330

Piacentino F.: B-631

Picano E.: B-123, B-324, B-325, B-326, B-523

Pienaar R.: $B-478$

Pieters-van den Bos I.C.: B-795

Pietilä A.-R.: B-577, B-580

Pietka E.: A-067

Pietrabissa A.: A-537

Pietsch H.: B-704, B-710

Pietschmann M.: B-567

Piffaretti G.: B-631, B-634

Pigneur F.: A-095, B-850

Pina Insausti L.J.: A-100

Pinggera G.: B-662

Pingitore A.: $B-207$

Pinilla I.: B-621, $B-622$

Pinol R.: B-199

Pinto A.: B-658

Pinto F.: B-658

Pirro F.: B-048, B-685
Pisano E.: $B-548$

Placier S.: $B-540$

Plagou A.: B-390

Platzgummer H.: B-604

Plumb A.A.O.: B-019

Plumhans C.: $B-128, B-636$

Poduri A.: B-478

Pogliani E.: B-055

Pohjola M.: B-580

Poiree S.: $B-814$

Poirier-Quinot M.: B-555

Polico I.: B-187

Pollice F.: B-637

Pollice P.: $B-637$

Pomerri F.: B-817

Pomier Layrargues G.: B-769

Pomper M.G.: A-175

Poncelet E.: B-796

Ponsioen C.Y.: $B-435$

Pontana F.: A-382, B-752, B-756

Poorbeigi $\mathrm{H}$ : : A-351

Poppert H.: B-291

Porfiri A.: $B-388$

Portalez D.: B-303

Porteous M.: B-741

Porto A.: B-285

Porto I.: B-094

Postma A.A.: B-087

Pöyskö H.: B-577, B-580

Pozzi Mucelli F.: $B-686$

Pozzi Mucelli R.: B-134, B-314, B-316, B-333, $B-496, B-797$

Pozzi-Mucelli R.: A-264

Prados C.: B-621, B-622

Prakken N.H.J.: B-340, B-830

Prassopoulos P.K.: A-508

Pravata' E.: $B-520$

Prayer D.: B-215, B-471, B-472, B-800, B-832, $B-833, B-834$

Preda L.: B-282, B-675

Pressney I.: B-013

Preux P.M.: B-059

Priego G.: $B-217$

Prior J.: $B-428$

Proctor I.: $B-436$

Profantova N.: $B-066$

Proietti A.: $B-187$

Prokop M.: A-025, A-253

Prosch H.: A-191

Pruessmann K.P.: A-336, B-570

Pruvo J.-P.: $A-121, B-043$

PS S.: $B-284$

Puderbach M.: B-626

Puderbach M.U.: A-499

Puesken M.: B-647

Puglielli E.: $B-353$

Pugliese S.: $B-108$

Puls R.: $B-131, B-224, B-383$

Punt C.J.A.: $A-343$

Punwani S.: B-436, B-522, B-739

Purpura P.: $B-103$

Püschel K.: B-620

Puylaert J.B.C.M.: A-317 


\begin{tabular}{l}
\hline \multicolumn{1}{c}{$\mathbf{Q}$} \\
\hline Qi Y.: B-239 \\
Qui P.-X.: B-557 \\
Qu H.: B-473 \\
Quaglio F.R.: B-477 \\
Quaia E.: B-001, B-166 \\
Quattrucci S.: B-624, B-625 \\
Quentin M.: B-601 \\
Quesada R.: B-038 \\
Quick H.H.: B-535, B-694 \\
Quintana M.: B-621, B-622 \\
\end{tabular}

Raatschen H.-J.: B-430

Racchiusa S.: B-493, B-791

Rácz A.: SS MS 1

Radaelli A.: B-156

Radbruch A.: B-214

Radeleff B.A.: B-257, B-260

Radke P.W.: B-208

Radkow T.: $B-593$

Ragab Y.: B-219, B-605

Rager O.: $B-772$

Ragot S.: $B-415$

Rahman N.: B-029

Rahmouni A.: A-095, B-850

Raible S.: $B-751$

Raimondi S.: B-009, B-025, B-675

Rainer S.: B-496

Rainford L.: B-444, B-571, B-573, B-574, B-808, $B-809$

Raininko R.: $B-831$

Rajpura H.: B-198

Rajput D.: B-616

Ramalho M.: B-539, B-619

Raman V.: B-332

Ramanna N.: B-284

Rampinelli C.: B-025

Rampoldi A.: B-590

Rana S.S.: B-254

Rancane B.: SS MS 4

Randi G.: B-028

Ranschaert E.R.: A-536

Rao N.: B-265, B-266

Rao P.: $B-156$

Rapezzi C.: B-203

Raptis D.: B-382, B-385

Raptopoulous V.: $B-256$

Rascioni M.: B-657

Rasekhi A.: A-349

Rasmus M.: $B-341$

Räßler F.: B-160

Räth C.W.: B-180

Ratib O.: A-069, B-772

Ratner E.: $B-189$

Ratti L.: $B-252$

Rau W.S.: B-640

Raupach R.: B-361, B-362, B-363, B-365, B-582, $B-853$

Ravanelli M.: B-196

Ravesloot C.J.: B-392

Raya J.G.: B-176

Raza S.: $B-431$

Reale C.A.: B-408

Rechichi G.: B-061

Recht M.: $B-176$

Reeder S.B.: A-384

Reekers J.A.: A-212

Regge D.: A-408, B-327, B-328

Regier M.: $B-623$

Regini F.: $B-482, B-483, B-485$

Reid J.H.: B-394
Reijnierse M.: A-083

Reil J.: $B-699$

Reimer P.: $B-345$

Reiner C.: B-382, B-385

Reiner C.S.: A-170

Reinhard M.: $B-688$

Reis R.B.: B-669

Reiser M.F.: A-446, B-050, B-074, B-097, B-160, $B-176, B-210, B-225, B-232, B-238, B-243$, $B-246, B-391, B-401, B-407, B-411, B-464$, $B-534, B-553, B-567, B-591, B-659, B-684$, $B-695, B-700, B-711, B-716, B-758, B-780$, $B-786, B-794, B-825, B-841, B-845, B-848$, $B-856 B-863$

Reitan A.: B-660

Reiter A.: B-052

Remírez J.: B-084

Rémy J.: A-382, B-274, B-752, B-756, B-757

Rémy-Jardin M.: A-382, B-274, B-752, B-756, B-757

Renate J.: $B-495$

Rengier F.: B-045, B-633

Rengo M.: $B-416$

Renker M.: B-753, B-755, B-759

Renz V.: $B-700$

Repa I.: B-579

Resch H.: B-179, B-789

Rescinito G.: B-677

Reske S.N.: B-022

Restaino G.: A-458, B-064, B-381

Rety F.: $B-116$

Revel M.-P.: A-326, B-395

Rey-Salmon C.: $A-037$

Reznek R.H.: A-024, A-109

Rhee H.: B-004

Ribeiro L.P.V.: B-443

Ribeiro M.M.C.P.: $B-441$

Ribet A.: B-093, B-209

Ricard F.: $B-116$

Ricart Selma V.: $B-317$

Riccabona M.: A-092, A-339

Ricci P.: $B-388, B-815$

Richard S.: $B-855$

Richards P.: $A-370$

Ridolfi F.: $B-613$

Riedl R.G.: B-169

Riemann J.F.: A-141

Riffel P.: B-052, B-342

Rigamonti P.: $B-525$

Righi V.: $B-617$

Righini A.: $A-228$

Rigou G.: B-670

Rimely E.: $B-635$

Rinaldi G.: B-203

Rinaldi P.: $B-687$

Ringelstein A.: $B-144$

Rios J.: B-586

Rischbieter P.: B-733

Ritschl L.: B-369

Ritz R.: B-216

Rivetti S.: $B-589$

Rix A.: $B-774$

Rizki M.: B-866

Rizzardi P.: $B-196$

Rizzatto G.: A-388

Rizzo A.: $B-658$

Rizzo S.: $B-282$

Robben S.G.F.: A-125, B-740

Robinson S.: A-287

Robson P.: B-531

Rockall A.G.: A-090, A-460

Rodrigues K.M.: B-478

Rodríguez C.: $B-115$

Rodriguez H.: B-621, B-622

Rodriguez-Justo M.: B-436
Rodt T.: B-537, B-778

Roemer F.W.: A-200, B-171, B-174, B-379, B-787

Rogalla P.: $A-248, B-258, B-331, B-339, B-821$

Roiati S.: $B-381$

Roig M.: $B-475$

Romani G.: B-520

Romano A.: B-108

Romano I.: B-498

Romano L.: B-658

Romano S.: A-304

Romeo R.: B-346

Romero N.: B-217

Rong D.: B-299

Rong R.: $B-164$

Ros M.: B-624, B-625

Ros P.R.: A-187, $A-430$

Rosenberg C.: $B-037$

Rosenblum M.: B-238

Rosendahl K.: A-452

Rossi A.: A-204, A-277, B-416, B-696, B-860

Rossini F.: $B-056$

Rostad E.: B-446

Roth C.: $B-190$

Roth $\mathrm{H} .:$ B-329

Rotondo A.: B-285, B-655

Rottbauer W.: $B-091$

Rousseau H.: A-534, B-043, B-414

Rovere-Querini P.: $B-781$

Rovira-Cañellas A.: A-390

Rowe G.: B-334

Rowe G.W.: B-412, B-497

Rowlands P.C.: A-066

Roycjowdhury A.: B-283

Rubin G.D.: B-044

Rubini A.: $B-563$

Rudas G.: B-738

Ruggiero A.: $B-771$

Ruimy D.: $B-606$

Rulseh A.: A-521

Rumboldt Z.: A-391

Rummeny E.: $B-100, B-419, B-552$

Rummeny E.J.: B-113, B-180, B-234, B-263, $B-291, B-305, B-348, B-376, B-421, B-484$, $B-603, B-861$

Rump J.: $B-510$

Runnebaum I.B.: B-549, B-643

Rusch O.: B-853

Russo R.: B-732, B-734

Russo V.: $B-203$

Rutten I.J.G.: B-169, B-528

Rutten M.: D-02

Rutten M.J.C.M.: B-075

Ryan A.G.: $A-467$

Ryan S.: A-127, B-612

Rydning A.: $B-660$

Ryeom H.-K.: B-846

Ryu Y.: $B-255$

\section{S}

Saad W.E.: B-767

Saake M.: $B-455$

Saam T: $A-325, B-243, B-246, B-464$

Saba L.: B-130, B-242, B-244, B-245, B-357

Saba O.: B-585

Sabatino F.: B-489

Sabharwal T.: A-466

Sabri S.S.: $B-767$

Sacconi B.: B-277

Sachs A.: SS MS 3

Sadick H.: B-199

Sadick M.: B-112, B-199

Sagar A.: B-254 
Sage C.: B-513

Sagot P.: $B-764$

Sahani D.: A-043, A-274

Saidi B.: $B-627$

Saini N.S.: $B-106$

Saito N.: B-358

Sakci Z.: B-515

Sakuma H.: B-092

Sala E.: A-042

Salega A.: $B-159$

Salemi S.: B-136, B-222

Salerno A.: $B-673$

Salgado R.: A-442

Salgarello M.: B-687

Salizzoni E.: $B-262$

Sallemi C.: B-205, B-781

Sallustio G.: B-064, B-381

Salomon L.J.: B-799

Salvador R.: A-048

Samei E.: B-855

Sánchez Reyes J.M.: B-086

Sanchez-Montañez A.: B-475

Sandner T.: $B-695$

Sandner T.A.: B-401

Sandolo F.: B-277

Sanfilippo R.: B-242, B-244, B-245, B-357

Sanghera B.: $B-120$

Sannia S.: $B-244$

Santangelo T.: $B-757$

Santini P.: $B-247$

Santoro M.: B-048, B-685

Santoro S.: B-146, B-687

Santos A.: B-450

Sanverdi E.: $B-211$

Saougou I.: B-276

Sarantidou K.: $B-736$

Sarchosoglou A.: A-397

Sardanelli F.: A-101, A-160, A-356, B-047, B-432, $B-501, B-525, B-541, B-828, B-864$

Sarno E.: $B-658$

Sarran A.: B-425

Sasiadek M.: B-213

Sassi C.: $B-262$

Saudek F.: A-344

Saueressig U.: B-073, B-598

Saugstad O.D.: $B-473$

Savarino E.: B-288, $B-432$

Savarino V.: $B-432$

Savelli S.: $B-731$

Savio D.: B-452

Saxena A.K.: B-827

Scandiffio R.: B-326

Scaranelo A.M.: B-286, B-320, B-866

Scarsbrook A.F.: B-194, B-527

Schaefer-Prokop C.: A-078, A-186, A-279

Schaefer-Prokop C.M.: B-392

Schaefers H.-J.: B-632

Schaeffeler C.: B-376

Schäfer A.-O.: B-018

Schäfers H.-J.: B-393

Schäffeler C.: B-603

Schännagel B.: B-206

Scharf J.: B-857

Scheffel H.: B-385

Scheffler K.: $B-178$

Schelhorn J.: B-487, B-679

Schell B.: B-697, B-753, B-755

Schellenberger E.A.: $A-471$

Scherer A.: B-248, B-601

Schernthaner R.: $B-129$

Scherr M.: B-856

Scherr M.K.: B-659

Scherrer A.: B-651

Schibler F.: B-751
Schillebeeckx J.: A-489

Schima W.: A-313, A-362

Schimpfke N.: B-706

Schindera S.T.: $B-575, B-751, B-853$

Schindler A.: B-243, B-464

Schlemmer H.-P.: A-457, B-135, B-148, B-719, B-867

Schmid M.: B-472, B-834

Schmidberger H.: B-530

Schmid-Bindert G.: B-023

Schmidt B.: B-365, B-382, B-582, B-585, B-853

Schmidt C.: $B-464$

Schmidt G.P.: B-160

Schmidt S.: B-427, B-428

Schmidt S.A.: B-022

Schmidt U.: B-480

Schmid-Tannwald C.: B-225, B-848

Schmitt B.: B-177

Schmitt P.: B-248

Schmitz C.: B-210

Schmitz-Rode T.: $B-749$

Schnackenburg B.: B-509, B-510

Schneider G.: B-699

Schneider G.K.: B-632, B-690

Schneider K.: B-591

Schoepf U.J.: B-238, B-334, B-412, B-413, B-468, $B-492, B-494, B-497, B-500, B-597, B-709$, $B-759, D-08$

Schoepf V.: B-588

Schönberg S.O.: A-227, B-023, B-052, B-112, $B-199, B-342, B-413, B-532, B-597, B-607$, $B-857$

Schöndube H.: B-368

Schönermarck U.: B-684

Schöpf V.: B-833

Schoth F.: $B-128$

Schotten S.: B-041

Schouman-Claeys E.: B-093

Schouman-Clayes E.: B-059

Schramm P.: B-102, B-105, B-359

Schramm R.: B-102, B-359

Schreer I.: $A-320$

Schreiber M.: B-690

Schreuder T.C.M.A.: B-384

Schreyer A.G.: B-085, B-440

Schuelke C.: B-647

Schueller-Weidekamm C.: B-179, B-604

Schuknecht B.F.: A-492

Schultze Kool L.: A-065

Schulze M.-K.: $B-149$

Schulz-Wendtland R.: B-802

Schurink G.-W.H.: B-344, B-350

Schwaiger M.: A-116, B-113, D-33

Schwartz L.: A-111

Schwarz B.: B-553, B-780

Schwarz F.: B-411, B-695, B-700, B-758, B-825

Schwarzwaelder C.B.: B-257, B-260

Schwier M.: B-649

Sciarra A.: B-308, B-666

Scofienza L.M.: B-373

Sconfienza L.M.: A-357, B-081, B-288, B-372, $B-432, B-501, B-742, B-864$

Scribano E.: $B-791$

Sebag G.-H.: A-154

Secchi A.: B-386, B-462

Secchi F.: $B-828$

Sedaghat A.: A-345

Segatto J.-M.: B-247

Segnan N.: $B-327$

Seidel R.: B-632, B-690, B-699

Seifarth H.: B-294

Seifert M.: $B-451$

Seimenis I.: $B-321$

Seitun S.: B-236, B-498

Semelka R.: B-539
Semelka R.C.: B-619

Sen M.: B-194

Senes F.M.: B-479

Senore C.: $B-327$

Seo B.K.: B-547, B-548

Seo H.I.: $B-490$

Seo H.S.: B-200

Seo J.M.: B-062, B-090, B-667

Seol H.Y.: $B-200$

Serafini G.: B-081, B-288, B-501

Seral P: : $B-084$

Serfaty J.-M.: B-093, B-209

Sergejeff J.: $B-577$

Sergi A.: $B-493$

Sergi M.E.: $B-693$

Serra G.: B-624, B-625

Sestieri $C$.: $B-520$

Settles M.: B-100, B-419, B-552

Setz-Pels W.: $B-805$

Sever A.R.: B-071

Seynaeve P.: $B-017$

Shabana A.: $B-561$

Shah S.: $B-198$

Shah V.: B-803

Shaheen E.: B-641

Shaikh S.: B-089

Shao H.: B-551

Sharma R.: B-132

Sharma S.: $B-497$

Shaterzadeh M.J.: B-268

Sheibani S.: A-351

Shen B.: B-715

Shen G.: B-370, B-859

Shen S.-H.: $B-683$

Shen Y.: $B-370$

Sheridan M.: B-426

Shi D.: B-297, B-360

Shibasaki J.: $B-476$

Shin B.K.: B-200

Shin H.J.: B-836

Shin J.K.: B-822

Shindoh J.: B-002

Shioyama Y.: $B-418$

Sho M.: B-133

Shokrollahi E.: B-268

Shuai X.-T.: B-557

Shukla-Dave A.: B-665

Siauve N.: B-799

Sidhu P.: B-612

Sidhu P.S.: $D-25, D-41$

Sidorenko I.: B-180

Siebelt M.: B-175

Siebenrock K.: B-378

Siebenrock K.A.: B-375

Sieber M.A.: B-704, B-710

Siegel M.: $D-09$

Siegmann K.: B-671

Siegmund W.: B-224

Siemund R.: $A-293$

Sieron D.: $B-155$

Sievert M.: B-603

Sigfridsson A.: B-092

Signorelli M.: $B-061$

Silakos A.: B-661

Silva C.A.: $B-443$

Silva C.I.S.: A-232

Silva M.: $B-028$

Silva-Fortes C.: B-441

Silverio R.: $A-242$

Silvestri E.: B-372, B-373, B-501, B-742

Silvestri R.: $B-279$

Simcock 1.: $B-803$

Simon D.: $B-135$

Simonetti G.: B-404, B-405, B-408, B-460, B-657 
Simonneau G.: B-420

Sindel T.: $B-768$

Singh M.: B-616

Singh N.K.: B-313

Sinnatamby R.: B-080

Siomou E.: B-736

Sirlin C.B.: $A-383$

Sironi S.: B-021, B-055, B-056, B-061, B-119, $B-162, B-252, B-253$

Sitbon O.: $B-420$

Sjövall K.: $A-398$

Skaane P.: A-099, B-186, B-725

Skogen K.: $B-013$

Skretting A.: B-473

Slabaugh G.: $B-329$

Slabu I.: $B-653$

Slooter G.: B-524

Slosman D.O.: $B-730$

Slowinski T.: B-078

Sluming V.: $B-519$

Smal Y.: $B-728$

Smaniotto D.: B-063

Smans K: A-496

Smeele L.E.: B-191

Smeets P.: $B-721$

Smith F.W.: $B-746$

Smith J.T.: B-426, B-664

Smith T.: $B-338$

Smits M.: A-051

Snider F.: B-048, B-685

Sobaszek A.: B-274

Sodhi K.S.: $B-827$

Sofikitis N.: B-661

Solano A.: B-602

Solbiati L.: A-157, B-290

Soldati C.: $B-462$

Sollazzo P.: $B-693$

Somma F.: $B-654, B-655$

Sommer C.M.: B-257, B-260

Sommer W.: B-050, B-232, B-411, B-758

Sommer W.H.: $B-391, B-841, B-845$

Son J.Y.: $B-822$

Song B.: $B-008$

Song J.Y.: B-200

Song M.G.: B-822

Sonoda L.I.: B-012, B-120

Soo K.C.: B-151

Soo K.C.C.: $B-152$

Sorantin E.: B-595

Sorrentino N.: $B-631$

Sosna J.: $B-858$

Soto J.A.: A-429

Sou $\mathrm{H}$.: $B-138$

Sourbron S.: B-210, B-841, B-845

Sourbron S.P.: $B-534$

Souza Jr. A.S.: A-233

Soylemezoglu F.: B-211

Soza G.: B-649

Sparacia G.: $B-103$

Spencer J.A.: A-040, A-081, A-505

Spieß J.: B-091

Spinelli A.: $B-408$

Spinnato P.: B-261, B-262

Spottiswoode B.: B-371

Sprigg A.: A-152

Squillaci E.: $B-657$

Srinivas M.: A-343

Srivastava D.N.: B-132

Staatz G.: A-498

Stäbler A.: A-372

Staehler M.: B-794

Stahl A.: $B-118$

Stajgis M.: A-162, A-481

Stampfl U.: B-257, B-260
Stanica C.: SS MS 2

Stanton A.: B-469

Stasik-Pres G.: B-104

Staudacher C.: B-014

Stavrakas P.: B-298

Stefanini M.: B-404, B-405

Steg G.: B-093

Steidle G.: B-536

Stein E.G.: B-586

Stein L.A.: B-121

Steiner H.: B-662

Steinert H.C.: $A-011$

Steingötter A.: $B-113$

Steinkamp H.J.: B-409

Stelzeneder D.: B-177, B-178, B-790

Stenberg L.: A-483

Stender G.: B-502

Stentarelli C.: B-615, B-617

Stern M.: B-651

Steward M.: B-436, B-522

Stieltjes B.: $B-135, B-214$

Stierstorfer K.: B-361, B-362, B-368

Stiller B.: B-598, B-599

Stiller W.: B-257, B-260, B-587

Stirling J.: $B-803$

Stivalet A.: B-796

Stocca T.: $B-686$

Stoeckel J.: B-188, B-189

Stojanović S.: B-595

Stojkovic M.: SS MS 1

Stoker J.: A-428, B-323, B-384, B-435, B-652, $D-17$

Stokkers P.C.F.: B-435

Stokroos R.J.: B-087

Stone J.R.: B-767

Storto M.-L.: A-281

Stosic-Opincal T.: B-195

Stotts G.: B-292

Stoupis C.: $A-507$

Strata E.: B-207

Strecker R.: $B-421$

Streitparth F.: B-509, B-510

Strobel K.: B-422

Stroobants S.: $A-272$

Stroszczynski C.: B-155

Strube P.: B-509

Struelens L.: B-726

Struffert T.: $B-455$

Studler U.: A-462

STUDY Group V.: B-223

Stumpp P.: B-007, B-149

Stylianou E.: B-051

Su X.: B-347

Sueyoshi S.: $B-133$

Sugawara Y.: $B-002$

Sugimura K.: $B-143, B-820$

Sugiura $H .: B-273$

Suh K.-S.: $B-611$

Suh S.: B-200

Suh Y.-L.: B-836

Suito $\mathrm{H} .: \mathrm{B}-044$

Sulzmann A.: B-148

Summers P.: B-009, B-481, B-675

Sun C.-H.: B-557

Sun G.: B-249, B-505, B-714

Sun H.Y.: $B-842$

Sun X.: B-715

Sun X.W.: B-164

Sun Y.-S.: B-016, B-816

Sun Z.: $B-551$

Sunaert S.: $B-101, B-513$

Sunami S.: $B-418$

Sundaram M.: A-149

Sundermann D.: B-724
Sundin A.: B-111

Sunnegardh J.: B-361, B-362

Suput D.: B-101

Suri J.S.: $B-130$

Suter A.: $B-377$

Suurküla M.: B-559

Svalkvist A.: $D-14$

Sverzellati N.: B-028, B-271, B-272

Swamy K.: B-284

Sweeney R.E.: B-494

Szczerbo-Trojanowska M.: A-117

Sze D.: B-044

Szewczyk P.: B-213

Szucs-Farkas Z.: B-575, B-751, B-853

\section{$\mathbf{T}$}

Tabarsi P.: B-627

Tacelli N.: B-757

Tachibana Y.: B-476

Taeger G.: B-051

Tagliafico A.: B-677

Tahmasebi M.: B-268

Tai D.C.S.: $B-389$

Taieb S.: B-425

Takahashi M.: B-002, B-358

Takahashi S.: B-820

Takase S.: B-092

Takenaka D.: B-143

Talasz H.: B-554

Talei Franzesi C.: B-061, B-162

Tali E.T.: A-251, A-518

Tan B.S.: $B-151$

Tan B.S.S.: B-152

Tan G.: B-761

Tan I.B.: B-191

Tan L.: B-514

Tanaka J.: B-581

Tanaka S.: B-543

Tanaka T.: B-133

Tanenbaum L.: B-586

Tang A.: B-769

Tang L.: B-016

Tang Y.: B-437, B-438

Tanter M.: B-540

Taouli B.: A-044

Tardaguila de la Fuente G.: B-233

Tardáguila Montero F.: B-233

Tardivon A.: A-352, A-431

Tarján Z.: A-302

Tarnoki A.D.: B-241

Tarnoki D.L.: $B-241$

Tartaro A.: B-489, B-520

Tasu J.-P.: $B-415$

Tatsch K.: A-059

Taule A.: B-442

Tavakoli B.: B-020

Tawfik A.: B-193

Tay K.H.: B-151

Tay K.H.H.: B-152

Taylor A.M.: A-107

Taylor K.A.: B-080

Taylor M.B.: B-019

Taylor S.: B-739

Taylor S.A.: A-142, B-329, B-330, B-436, B-522

Tchouante P.: $B-261$

Teertstra H.J.: B-191

Teichgräber U.: B-509, B-510

Telesca M.: B-544, B-546, B-676, B-678

Temel O.: B-515

ter Haar Romeny B.M.: A-449

Terlizzi A.: B-631

Thamm P.: B-131 
Theisen D.: B-097

Theotokas I.: B-390

Thiam R.: B-799

Thibault J.-B.: B-364

Thieme M.E.: B-652

Thieme S.F.: B-391

Thierens H.: B-721

Thierfelder C.: $B-148$

Thilo C.: B-238, B-500

Thng C.H.: B-151

Thoeny H.C.: $B-538$

Thomas A.: $B-078, D-23$

Thomassin-Naggara I.: A-041, B-796

Thorolfsdottir A.: A-396

Thron A.K.: A-524

Thürmel K.: B-603

Thurner G.C.: $B-554$

Thurnher M.M.: A-050, A-244

Tianging C.: $B-023$

Ticca C.: B-590

Tiddens H.A.W.M.: B-592, B-624, B-625

Tietjen C.: $B-649$

Tilea B.: A-154

Tinelli G.: B-685

Tins B.: A-371

Tintera J.: $A-521$

Tisserand M.: $B-456$

Tobajas Morlana P.: B-038

Todd-Pokropek A.: A-068

Todua F.I.: B-354

Togashi K.: B-543

Tolooee S.: $A-351$

Tomà P.: A-094, B-732, B-734

Tomandl B.: B-293

Tomei A.: B-693

Toms A.P.: B-433, B-434

Tondolo T.: $B-290$

Torkzad M.R.: B-735

Torner J.: B-171

Torresin A.: B-590

Torricelli P.: B-117, B-202, B-615, B-617

Tosi G.: B-047

Tóth Z:: B-738

Touzé E.: B-456

Traen S.: B-017

Tramalloni J.: B-814

Tramontin S.C.M.: A-231

Trampal C.: B-114

Tran-Van-Nhieu J.: B-850

Trasimeni G.: B-108

Trattnig S.: A-257, B-173, B-177, B-178, B-745, $B-790$

Treanor D.: B-426

Treece G.M.: B-080

Treier R.: B-575

Treitl M.: B-401, B-407, B-856

Tresoldi S.: B-240

Treumann T.: $B-422$

Triantafyllou M.: $B-538$

Trimboli R.M.: B-047, B-541

Tritella S.: $B-864$

Trojanowska A.: A-224

Tropres I.: B-512

Trueb P.R.: B-575

Trumm C.: $B-160$

Tsalikis M.: B-298

Tsampalas S.: $B-661$

Tsampoulas C.: B-736

Tsampoulas K.: B-276, B-661

Tsavalas N.: B-782, $B-784$

Tscholl D.: B-632

Tsili A.C.: B-661

Tsitas K.: $B-466$

Tucci Jr S.: B-669
Turba U.C.: B-767

Turgut A.T.: $A-422$

Turmezei T.D.: $B-566$

Turowski B.: B-248

Tüttenberg J.: $B-110$

Tychyj-Pinel C.: $B-116$

Tzedakis A.: B-321

Tzoanos G.: B-784

$\overline{\mathbf{U}}$

Uder M.: B-124, B-293, B-593, B-802

Udo K.: $B-302$

Ueda T.: $B-044$

Uhl M.: B-659, $B-688$

Uhlenbrock D.: $D-01$

Uitterdijk A.: B-696

Ulcigrai V.: $B-166$

Ullah E.: B-608

Ullberg C.: A-216

Ullberg U.: B-735

Umeda I.: $B-776$

Umutlu L.: B-051, B-118, B-486, B-535

Ungureanu G.: SS MS 2

Unterhinninghofen R.: B-045

Urbach H.: B-451

Uren N.: B-338

$\overline{\text { V }}$

Vadvala H.V: B-198

Vafeiadis E.: $B-390$

Vag T.: $B-318, B-550$

Vagli P.: $B-123, B-324, B-325, B-326, B-523$

Vahl C.-F.: $B-041$

Válek V.: A-303, A-374

Valentini A.: B-063

Valentinitsch A.: B-127, B-179

Valette P.J.: $B-116$

Valette P.-J.: $D-18$

Valkema R.: $B-812$

Valle M.: $B-479$

Vallee J.-P.: $B-772$

Vallerio P.: $B-496$

Vallet F.: $B-415$

Valsecchi M.G.: B-061

van Beek E.J.R.: B-338, B-394

van Beek M.: B-806

Van Beers B.: A-386

van Breest Smallenburg V.: B-805, B-806

van Buchem M.A.: B-351, B-352

Van Cauter S.: B-101

van Dam R.M.: B-167

van Delden O.M.: A-418

van den Berk I.A.H.: B-392

van den Bosch H.C.: B-671

Van den Hauwe L.: A-295, A-436, A-523

van der Giessen W.J.: B-696

van der Grond J.: B-351, B-352

van der Lugt A.: $B-812$

Van der Zijden T.: A-295, A-436, B-458

van Dijke C.F.: $B-556$

van Eijck C.: $B-812$

van Engen R.E.: $B-723$

van Gelder R.E.: B-323

Van Goethem J.W.: A-295, A-436

Van Goethem J.W.M.: A-523

Van Gompel G.: B-851

Van Gool S.: B-101

Van Hecke W.: B-513, B-517

Van Hoof T.: B-721

van Kuijk C.: B-529, B-795
Van Ongeval C.: $B-641$

van Overhagen $\mathrm{H}$.: A-297

van Rijn R.R.: A-451

Van Rooy F.: B-017

van Straten M.: B-175, B-592, B-696, B-860

van Tiel J.: B-175

van Velthuysen M.-L.F.: B-191

van Vliet M.: B-556

van Waesberghe J.H.H.T.M.: B-529, B-795

van Werven J.R.: B-384

Vande Berg B.: A-199

Vande Berg B.C.: B-563, B-564

Vandecaveye V.: A-088

Vandulek C.: B-579

Vanhoenacker F.M.H.M.: A-415

Vaño M.: B-154, B-317

Vansteenkiste F.: B-017

Vargas da Silveira Cunha Cruz V.C.: B-749

Vargas H.A.: B-302, B-665

Vassalou E.: $B-782, B-784$

Vasselli F.: B-544, B-546, B-676, B-678

Vaudano G.P.: B-452

Vaz G.E.I.: B-441

Vázquez E.: A-278, B-475

vd Brekel M.W.M.: B-191

Vedel B.: B-068

Vee B.: $B-442$

Veenland J.F.: B-556, B-560

Vega M.L.: B-115

Veillon F.: A-061

Veit-Haibach P.: B-422

Velonakis G.: B-503, B-506

Veloza L.-S.: $B-587$

Velthuis B.K.: B-340, B-830

Veltman J.: $A-161$

Vemmou A.: B-298

Venancio J.: A-420

Venkatesh S.K.: B-389

Venneri P.: $B-663$

Venstermans C.: A-295, A-436, A-523

Ventriglia F.: $B-693$

Venturini M.: $B-386, B-462$

Venugopal V.: $B-608$

Vera A.: B-154

Verardi N.: $B-541$

Verbist B.: A-062

Verdonk P.: B-785, B-788

Verhaar J.A.N.: B-175

Verhulst M.L.: B-524

Verschakelen J.A.: A-280, A-472

Versluis B.: B-344, $B-350$

Versluis M.J.: $B-351, B-352$

Verstraete $\mathrm{K}$ : $A-377, B-721, B-785, B-788$

Vessal K.: A-348, A-350

Veyrac C.: A-338

Viallon M.: B-772

Vidmar Kocijancic K.: A-167

Vieira R.: B-567

Vikgren J.: $D-14$

Vilanova J.C.: $A-414$

Vilar J.: A-503

Vilela P.: $A-438$

Vilgrain V.: A-097, B-425

Villa A.: $B-677$

Villa F.: B-240, B-525

Ville Y.: $B-799$

Villeirs G.M.: B-307

Villien M.: B-512

Vinci V.: $B-693$

Viotti S.: B-009

Virgili G.: B-482

Virmani S.: B-322

Vlahos K.: B-276

Vliegenthart Proença R.: B-280 
Vock P.: A-291, B-575, B-751, B-853

Vogl T.: $B-779$

Vogl T.J.: $B-031, B-032, B-033, B-034, B-035$ $B-036, B-067, B-147, B-158, B-159, B-193$, $B-289, B-423, B-424, B-445, B-502, B-697$, $B-737, B-753, B-755, B-759, D-19$

Vöglein J.: $B-110$

Vogt F.M.: B-208, B-807

Voillemot N.: $B-814$

Vollmann R.: $B-250$

Volterrani L.: $B-650$

Völzke H.: B-131, B-383

von Allmen G.: $B-575$

Von Einem J.: B-553, B-780

von Keudell A.G.: $B-789$

von Schulthess G.K.: $D-07$

von Kummer R.: A-294

von Tengg-Kobligk H.: B-045, B-633, B-867

Vonken E.P.A.: B-824

Voogd A.C.: $B-806$

Voormolen M.: A-295, A-436

Voormolen M.H.J.: B-458

Vorwerk D.: A-358

Votrubová J.: A-155

Vrabec M.: B-101

Vuong T.: $B-526$

Vymazal J.: A-521

\section{W}

Waarsing J.H.: B-175

Wagel J.: $B-213$

Wagner A.A.: B-531

Wagner J.: $B-583$

Wagnetz U.: B-629

Wahab S.: $B-608$

Wakhloo A.: B-502

Walcher D.: B-091

Walcher T.: B-091

Waldt S.: A-084, B-603

Wallace W.A.: $B-566$

Wallis M.G.: A-098, B-080, B-185, B-801

Wallner C.-P.: $B-591$

Wallnöfer E.A.: B-554

Walsh S.L.F.: B-271, B-272

Walter T.: $B-510$

Wang D.: B-336, B-715

Wang H.: B-429, B-514, B-775

Wang K.: $B-171, B-174$

Wang M.: B-297, B-360, B-454

Wang Q.: B-145, B-387

Wang R.P.: $B-046$

Wang T.: $B-763$

Wang W.L.Q.: B-270

Wang X.: B-596, B-826

Wang X.-F.: B-775

Wang X.M.: B-840

Wang X.Y.: $B-164$

Wang Y.: $B-280$

Wang Z.: B-508

Wang Z.-L.: B-016

Ward J.: B-426

Ward M.D.: A-220

Wardlaw D.: B-746

Warnking J.: $B-512$

Wassberg C.: B-665

Watadani T.: $B-358$

Watanabe R.: $B-476$

Webb A.G.: B-351, B-352

Weber C.: B-057, B-058, B-467

Weber G.: B-179

Weber M.: B-215, B-471, B-604, B-800

Weber M.-A.: $B-110$
Weber O.: B-096

Weber T.F.: $B-633$

Weber W.: A-112

Weckbach S.: B-050, B-246, B-607

Wedegärtner U.: B-600

Wee A.: B-389

Weeks J.: $B-071$

Weibrecht M.: B-128

Weigel S.: $B-182$

Weimer M.: B-110

Weinans $\mathrm{H}$ : : $B-175$

Weinheimer O.: $B-626$

Weininger M.: B-334, B-412, B-413, B-709

Weir N.: B-338

Weishaupt D.: A-169

Weiss M.: $B-681$

Weisser P.: B-753

Weitschies W.: $B-224$

Weller J.: B-706

Wells A.U.: B-271, B-272

Welsch G.H.: B-173, B-178, B-790

Wen L.: B-713

Wen Z.: B-347

Wendel F.: B-656

Wenkel E.: B-802

Werlen S.: B-375, B-378

Werner H.: B-692

Werner S.: B-790

Wessely M.: B-684

Wessling J.: B-647

West D.J.: A-307

Westphalen A.C.A.: B-669

Wetzels J.: $B-705$

Whitby E.H.: A-236

Wiarda B.M.: B-652

Wibmer A.: B-844

Wick W.: $B-214$

Widhalm G.: B-215

Wielpütz M.O.: B-626

Wießpeiner U.: $B-250$

Wiestler B.: B-214

Wiggermann P.: B-155

Wijeratna M.: $B-741$

Wikström J.: B-831

Wilcox C.: B-256

Wildberger J.E.: B-344, B-350

Wildgruber M.: B-552

Wilhelm C.: $B-555$

Wilkens H.: B-393

Wilkinson V.: $B-400$

Willi J.-P.: $B-772$

Williams M.C.: $B-338$

Willinek W.A.: $B-451$

Wilmink J.T.: A-524

Wilms G.: A-181, A-250

Wilson D.: $B-426$

Wilson D.J.: A-261, B-747

Wilson R.B.: B-497

Windfuhr-Blum M.: B-073

Winter L.M.: B-096

Winterhalder R.: B-422

Wintersperger B.: B-097, B-695

Wintersperger B.J.: A-054, B-553, B-780

Winterstein A.: B-379

Wirth B.: D-06

Wirth M.: B-690

Wirth S.: B-659, B-716, B-856, B-863

Wissgott C.: B-409

Wittenberg R.: B-392

Wittens C.: B-636

Wittsack H.-J.: B-601

Woertler K.: B-376

Wöhrle N.: B-074

Woitek R.: B-471
Wolf $\mathrm{H} .:$ B-585

Wolf K.-J.: $B-430$

Wolf M.: B-702

Wong K.: $B-337$

Wong L.F.: B-394

Wong W.K.R.: B-852

Wong W.L.: $B-120$

Wonneberger U.: B-510

Woo O.H.: B-547

Woodcock T.: $B-335$

Wörtler K.: A-515, B-603

Wu C.-L.: $B-683$

Wu J.: $B-698$

Wu L.: B-596, B-610

Wu Q.: $B-453$

Wu Y.: $B-437, B-438$

Wu Z.Y.: $B-514$

Wulfert S.: B-706

Wyatt J.: B-426

Wyss M.: B-570

Wyttenbach R.: B-247

$\mathbf{X}$

Xiao X.-S.: $B-157$

Xiao X.-S.: B-141

Xie S.: $B-336$

Xie X.: $B-040, B-280$

Xu J.Q.: B-545

Xu K.: $B-551$

Xu L.: B-614

Xu N.: B-235

Xu R.: $B-170$

Xu S.: $B-259, B-389$

Xu W.J.: $B-783$

Xu Y.: $B-142, B-713$

Xyda A.: B-102

$\overline{\mathbf{Y}}$

Yabuuchi H.: B-418

Yaffe M.J.: $A-268$

Yagil Y.: $B-398$

Yakimov A.O.: $B-838$

Yamaga L.: B-583

Yamaguchi M.: $B-776$

Yamamura J.: B-206, B-600

Yamashita K.: B-107

Yang J.: B-761

Yang J.-Y.: B-557

Yang L.: B-698

Yang Y.J.L.: B-270

Yang Y.Z.Y.: B-270

Yang Z.G.: B-011, B-030

Yang Z.-G.: B-521

Yap S.P.: B-264

Yasunaga K.: B-274

Ye X.-D.: B-157

Ye Z.-X.: B-429

Yerli H.: B-079

Yi N.-J.: B-611

Yilmabasar M.G.: B-744

Yilmaz S.: $B-768$

Yilmaz T.: B-079

Yin G.: B-098

Ying $Y .: B-145$

Yoo J.L.: B-200

Yoo J.Y.: B-499

Yoo S.-Y.: B-836

Yoon J.-Y.: B-410

Yoon Y.: B-092

Yoong P.: B-741

Yorozuya K.: B-804 
Yoshida H.: B-328

Yoshida M.: B-804

Yoshikawa T.: B-143, B-820, B-868

Yoshiura T.: B-107

You C.: B-514

Young D.: $B-743$

Young K.C.: $B-723$

Yousry T.A.: A-049

Yu C.: $B-518$

Yu H.: B-389

Yu J.-S.: B-792, B-847

Yu S.K.: $B-852$

Yu T.: $B-145$

Yuan C.: B-243, B-464

Yuan Y.: B-065

Yuan Z.: B-157

Yun I.J.: B-639

\section{Z}

Zaccagna F.: B-049, B-349, B-488, B-491, B-507

Zaccarella A.: B-797

Zachrisson S.: $D-14$

Zagdanski P.: B-188

Zagvozdkin E.: SS MS 4

Zahirifard S.: B-627

Zak L.: B-173

Zaloni E.: SS MS 2

Zanca F.: B-185

Zander T.: B-422

Zanello A.: $B-150$

Zanetti M.: A-193, B-377

Zangos S.: B-031, B-159, B-289

Zarb F.: B-571, B-573

Zareski E.: $A-159$

Zbyn S.: B-177, B-178

Zearo R.: $B-315$

Zech C.: B-841, B-845

Zech C.J.: A-265, A-300, B-223, B-225, B-848

Zechmann C.M.: B-671

Zedenius J.: $B$-111

Zellweger M.: B-096

Zeng D.: B-548

Zeng K.: B-364

Zengel P.: $B-246$

Zhang G.: B-370, B-763

Zhang G.-X.: $B-775$

Zhang $\mathrm{H}$.: $B-715$

Zhang J.: B-453, B-551, B-665

Zhang J.W.: $B-220$

Zhang L.: B-145, B-235, B-454, B-508, B-754, B-760

Zhang M.: B-299

Zhang Q.: B-336

Zhang S.: $B-336$

Zhang X.-P.: B-016, B-798, B-816

Zhang X.-Y.: B-798, B-816

Zhang Z.: B-470, B-495

Zhao J.-L.: $B-775$

Zhao S.: B-098, B-691

Zhao X.: $B-618$

Zhao Y.E.: $B-754$

Zheng J.: B-302

Zheng L.: B-283

Zheng L.-F.: $B-775$

Zheng X.: B-370

Zheng Y.: B-508

Zhong X.: $B-371$

Zhou C.: B-454, B-754, B-760

Zhou L.: B-304

Zhu N.: B-618

Zhu R.: B-320

Zhuang H.: B-521

Ziech M.L.W.: B-435
Ziegeler E.: B-659

Ziegler S.: $A-240$

Zienhom F.: B-219

Zilkens C.: $B-601$

Zimbaro F.: B-493, B-791

Zimny A.: B-213

Zimolo Y.: $B-686$

Zini C.: $B-049$

Zins M.: A-183, A-314, A-506

Zogbi-Neto O.: $B-669$

Zompatori M.: B-028

Zona S.: B-617

Zou K.H.: B-709

Zou Q.: B-429

Zoubi R.: B-318, B-549, B-550, B-643, B-644

Zoumpoulis P.S.: $B-390$

Zourla A.: B-298

Zsbán M.: B-738

Zscharn M.: B-786

Zsivcsec B.: B-430

Zubarev A.: B-069

Zucconi F.: B-590

Zucman-Rossi J.: B-850

Zuehlsdorff S.: $B-098$

Zuiani C.: B-072, B-311, B-312, B-315

Zwerner P.L.: B-238 